\title{
Expanding or Static Universe: Emergence of a New Paradigm
}

\author{
Paul A. LaViolette \\ The Starburst Foundation, Niskayuna, NY, USA \\ Email: plaviolette@starburstfound.org
}

How to cite this paper: LaViolette, P.A. (2021) Expanding or Static Universe: Emergence of a New Paradigm. International Journal of Astronomy and Astrophysics, 11, 190-231.

https://doi.org/10.4236/ijaa.2021.112011

Received: March 11, 2021

Accepted: May 28, 2021

Published: June 1, 2021

Copyright $\odot 2021$ by author(s) and Scientific Research Publishing Inc. This work is licensed under the Creative Commons Attribution International License (CC BY 4.0).

http://creativecommons.org/licenses/by/4.0/

(c) (i) Open Access

\begin{abstract}
The no-evolution, concordance expanding universe cosmology and no-evolution, static universe tired light model are compared against observational data on eight cosmology tests. The no-evolution tired light model is found to make a superior fit on all tests. Any attempts to introduce evolutionary corrections to improve the concordance cosmology fit on one test often worsen its fit on other tests. Light curve data of high redshift gamma ray bursts and quasars fail to support claims for cosmological time dilation due to expansion. Also, the SCP supernova light curve test results are considered to be flawed by selection effect biases. The big bang theory also has difficulty accounting for redshift quantization, for the multi-megaparsec periodicity seen in the distribution of galaxy superclusters, and for the discovery of galaxies at redshifts as high as $z \sim 11.9$. In overview, it is concluded that a static universe cosmology must be sought to explain the origin of the universe. One possible choice is a cosmology that predicts nonconservative tired-light redshifting in intergalactic space, the continuous creation of neutrons in space, the rate of matter creation scaling with both celestial body mass and temperature, galaxies growing progressively in size, and changing their morphology in the manner suggested by Jeans and Hubble.
\end{abstract}

\section{Keywords}

Cosmological Redshift, Tired Light Effect, Hubble Constant, Galactic Evolution, Continuous Creation, Subatomic Particles, Reaction-Diffusion Systems, Open Systems, Self-Organizing Systems, Observational Cosmology, Tolman Test, Angular-Size-Redshift Test, Hubble Diagram, Supernovae

\section{Introduction}

In the past, there have been two main interpretations of the cosmological red- 
shift phenomenon, the standard interpretation asserting that it is a recessional cosmological effect due to the expansion of space-time, and the competing view that the universe is not expanding and that the redshift is instead due to a "tired light" energy loss that photons undergo on their journey through space. These two models are most equitably compared to cosmological test data by refraining from introducing ad hoc evolutionary corrections. That is, the discrepancy of each model relative to the data trend becomes most apparent in the absence of evolutionary adjustments. This should not be taken to imply that galaxies do not evolve over time. Indeed, evolution is expected to occur in both the conventional big bang cosmology and to a lesser extent in the static universe, tired light cosmology since the latter does not have the same time limitations for the beginning of creation. Nevertheless, due to the uncertainty in knowing beforehand how much evolution actually would take place in each competing cosmology, it is best to entirely avoid making evolutionary assumptions and to choose the no-evolution model that makes the best fit to the data.

In the next section, the no-evolution, static universe tired light hypothesis and the no-evolution, expanding universe hypothesis are compared against data on several cosmology tests: the angular-size-redshift test, the galaxy number-count-magnitude test, the Tolman surface brightness test, the Hubble diagram test, and its variation the photon-flight-time-redshift test. As will become apparent, the use of multiple tests to compare competing cosmologies is more than just a review of past comparisons. It is an interactive approach to cosmology testing wherein a cosmological model's performance on any given cosmology test is made accountable to its performance on the other tests being examined. Thus, any assumptions introduced to the no-evolution prediction of a given cosmology with the intention of allowing it to better fit one set of test data must be applied as constraints to the interpretation of that cosmology on the other cosmology tests. It is found that in many cases, assumptions applied to make a cosmology fit better on one test, worsen its fit on another test. A final judgment as to the superiority of one cosmology over the other is made by considering the cosmology's performance on all tests, rather than on any one specific test in isolation from the others.

An overview of Section 2 shows that the no-evolution tired light model makes the best fit to the data on all tests without the introduction of ad hoc assumptions. The no-evolution expanding universe cosmology, on the other hand, is able to fit all the data only if numerous ad hoc assumptions are introduced specifying major evolution in galaxy cluster size, galaxy angular size, galaxy radio lobe size, galaxy luminosity, galaxy surface brightness, and galaxy number density. This however raises the question as to why cosmologists should stick with the expanding universe hypothesis with its need of ad hoc corrections chosen in such a way as to allow it to fit the various data trends, when the tired light model already fits the data reasonably well on all tests without making such adjustments. Thus, the tired light cosmology is found to be preferred because of its overall simplicity. 
Finally, Section 3 examines the validity of past claims for the occurrence of time dilation in distant supernova since such claims play a critical role in the assumption of whether the universe is static or expanding. Also, in that section we examine the implications of the redshift quantization phenomenon in regard to the choice of the static universe or expanding universe alternatives. In addition, other difficulties for the big bang alternative are examined such as the multi megaparsec supercluster periodicity and the existence of galaxies at high redshift. Section 4 examines the conservative and nonconservative tired light model alternatives and whether it may be necessary to consider that our universe operates at a fundamental level as an open system allowing small departures from perfect energy conservation. Section 5 examines continuous matter creation cosmologies as a possible substitute for the failed big bang paradigm.

\section{Testing the Competing Cosmologies}

\subsection{The Angular-Size-Redshift Test}

The first cosmological test to be considered is the angular-size-redshift test. In this version of the test, distance is judged based on the angular size, $\theta$, which is derived by observing the angular separations between bright galaxies in a cluster, as seen projected on the plane of the sky, and calculating the corrected harmonic mean of these separations. This $\theta$ value is then plotted against the cluster's redshift. One suitable $\theta-z$ data set is that published by Hickson and Adams [1] for a set of 94 galaxy clusters and which includes clusters at moderately high redshifts reaching up to $z=0.46$. Figure 1 is adapted from the paper of LaViolette [2] who had plotted the linear Hubble relation, the no-evolution, static universe

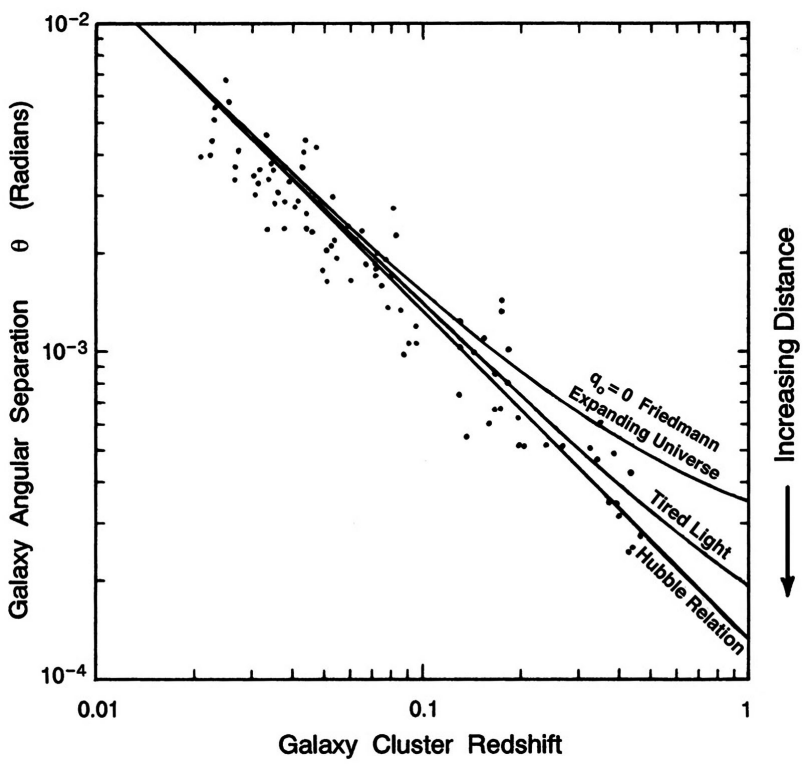

Figure 1. Harmonic mean angular separation for the brightest galaxies in a cluster plotted vs. redshift for 94 galaxy clusters. The no-evolution tired light model makes a far better fit to the data than the no-evolution $q_{0}=0$ Friedmann model assuming universal expansion. (After LaViolette [2] using data from Hickson and Adams [1]). 
tired light model, and the no-evolution, expanding universe $q_{0}=0$ Friedmann model against the Hickson-Adams data set. LaViolette assumed an $H_{0}$ of 55 $\mathrm{km} / \mathrm{s} / \mathrm{Mpc}$, as did Hickson and Adams. While this choice of $H_{0}$ is somewhat low compared to the range of currently published values, it does not influence the test outcome since both data and models are similarly affected. Also, while the data for this one test is relatively old, dating from 1979, no newer data on the mean angular separation of galaxies in a cluster has become available. Moreover, since the results of the cosmology comparison performed by LaViolette have passed the test of time, we feel this test should be included here.

The Hubble relation, which assumes a linear $r-z$ variation, $z=H_{0} r / c$ for Euclidean space, predicts that cluster angular size $\theta$ should vary with distance $r$ as:

$$
\theta=d_{o} / r=k / z
$$

where $k=d_{o} H_{0} / c$ and $d_{o}=0.75 \pm 0.15 \mathrm{Mpc}$ is the intrinsic size determined for a typical cluster. This is plotted in Figure 1 as the downward sloping straight line.

The tired light model specifies that a photon loses energy during its journey through Euclidean space as:

$$
E(r)=E_{0} \mathrm{e}^{-\beta r}
$$

where $\beta=H_{0} / c$ represents the rate of energy attenuation and $\mathrm{r}$ the distance traveled. This implies that photon wavelength $\lambda$ should increase exponentially with distance as:

$$
\lambda(r)=\lambda_{0} \mathrm{e}^{\beta r}
$$

and that photon redshift, $Z$, should vary with distance as:

$$
z=(\lambda(r)-\lambda) / \lambda_{0}=\mathrm{e}^{\beta r}-1
$$

where $\lambda_{0}$ is the wavelength of the photon at the time of emission. This in turn yields:

$$
r=\ln (1+z) / \beta
$$

Hence if no cluster evolution is assumed, the static universe, tired light relation predicts that cluster size should vary as:

$$
\theta=k / \ln (1+z) \text {. }
$$

This appears in Figure 1 as the slightly curved solid line, diverging slightly upward from the linear Hubble relation. For small propagation distances, $\beta r \ll 1$, Equation (6) may be approximated by the linear relation, Equation (1).

The no-evolution Friedmann model having a $q_{0}=0$ deceleration parameter and a $\Lambda=0$ cosmological constant, which is here chosen as the expanding universe alternative, predicts that cluster size should vary as:

$$
\theta=\frac{k(1+z)^{2}}{z(1+z / 2)}
$$

where $k$ is the same as for Equation (6). This is plotted in Figure 1 as the upper most curved solid line. Based on observational evidence, Gott, et al. [3] have 
proposed that $q_{0}=0.09$ for a Hubble constant of $65 \mathrm{~km} / \mathrm{s} / \mathrm{Mpc}$, and in their mass density study Bahcall and Fan [4] have proposed $q_{0}=0.1 \pm 0.5$. So, it is reasonable to choose the $q_{0}=0$ Friedmann model for comparison to the tired light model since the $q_{0}=0.1$ model prediction deviates only slightly.

Assumption-laden Friedmann models with larger $q_{0}$ values, such as the $q_{0}$ $=0.5$ model, would plot substantially above the $q_{0}=0$ cosmology and would depart even further from the data trend. The more commonly cited $\Lambda \mathrm{CDM}$ cosmology with $\Omega_{\mathrm{M}}=0.3$ and $\Omega_{\Lambda}=0.7$, which Goldhaber, et al. [5] had used in interpreting their supernova data (discussed in Section 3), when plotted on this test using $H_{0}=55 \mathrm{~km} / \mathrm{s} / \mathrm{Mpc}$, is virtually indistinguishable from the $q_{0}=0$ model over this redshift range. Hence there is no point to plot it.

LaViolette [2] compares the fit of these three models, by assessing the variances between the $\theta$ data points and the prediction each model makes. He finds that variances for 1) the linear $\theta \propto 1 / z$ relation, 2) the tired light model, and 3) the expanding universe model compare respectively in the ratio 1:1.2:5.0. Repeating the calculation for the 31 most distant clusters $(z>0.1)$ gives relative variance ratios of 1:1.4:10. Thus the static, Euclidean tired light cosmology is seen to be significantly favored over the $q_{0}=0$ expanding universe model, requiring no need to introduce ad hoc assumptions about cluster size evolution.

Lopez-Corredoira [6] has performed a study of the angular radii of galaxies over the redshift range $z=0.2$ to 3.2 and also concludes that the angular sizes for galaxies conform more closely to a static, tired light cosmology with no need to assume size evolution. In his comparison of cosmologies, he includes the no-evolution tired light model and the no-evolution concordance cosmology $\left(H_{0}\right.$ $\left.=70 \mathrm{~km} / \mathrm{s} / \mathrm{Mpc}, \Omega_{\mathrm{M}}=0.3, \Omega_{\Lambda}=0.7\right)$. The comparison is insensitive to the choice of $\mathrm{H}_{0}$. It was necessary for Lopez-Corredoira to use separate graphs for comparing each cosmology to his data set since his distance determinations are galaxy luminosity dependent, which in turn are sensitive to the particular cosmology being assumed. Figure 2, adapted from Figure 2 of his paper, shows the results

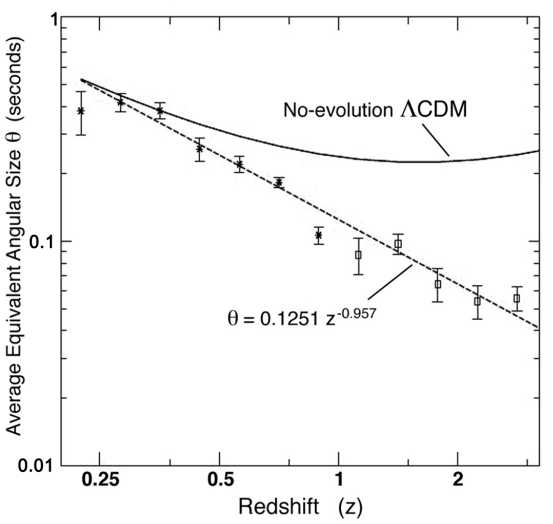

(a)

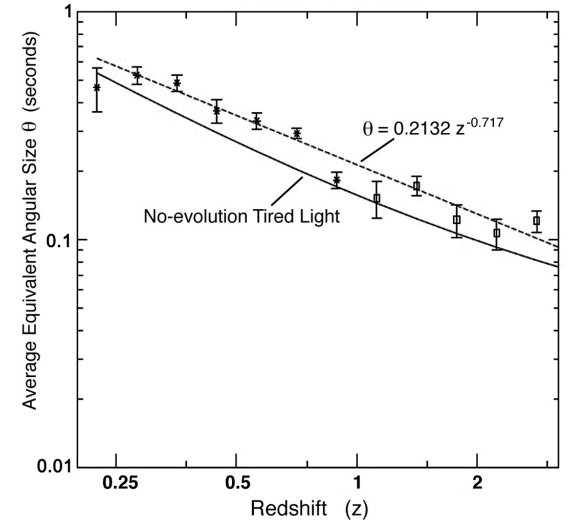

(b)

Figure 2. Comparison of alternative cosmologies to data on the galaxy angular size-redshift test, (a) the no-evolution $\Lambda \mathrm{CDM}$ expanding universe cosmology and (b) the no-evolution tired light static universe cosmology. 
obtained when comparing against galaxy angular size-redshift data: a) the no-evolution concordance cosmology and b) the no-evolution tired light cosmology. As is apparent, the tired light cosmology more closely follows the angular size-redshift data trend (dotted line). So, as in the previous angular-size redshift test, this test also demonstrates that the tired light model makes a superior fit, but using an entirely different angular statistic, namely galaxy angular size rather than the angular separation of galaxies in a cluster. If plotted in Figure 2(a), the $q_{0}=0$ Friedmann model would track close to the $\Lambda$ CDM cosmology, but for $z>1.5$ would begin to depart somewhat below its trend line.

It is noteworthy that the data trend throughout its redshift range is consistently offset below the tired light prediction by about the same amount over all redshifts, indicating galaxy diameters slightly larger than expected. Lopez Corredoira [6] attributes this to the possibility of dust extinction which affects the luminosity dependent method used in determining galaxy distances for the data set. Figure 3, adapted from Figure 7 of his paper, shows that this gap is closed by assuming a dust extinction of $a_{V}=3.4 \times 10^{-4} \mathrm{Mpc}^{-1}$, which is equivalent to assuming an intergalactic dust density of $\rho_{\text {dust }} \sim 1.2 \times 10^{-33} \mathrm{~g} / \mathrm{cm}^{3}$. It is proposed in Section 2.3 below that dust extinction also plays an important role in the Tolman test.

Yet another type of angular-size-redshift test utilizes, as the angular size, radio lobe separation indouble-lobed radio galaxies and quasars [7] [8]. Ubachukwu and Onuora [9] conducted one such test which compares quasar data extending up to $z=2.1$ to various cosmological models; see Figure 4. They conclude that the static-universe, tired light model gives the best fit. The most favorable no-evolution Friedmann model which assumes a minimally curved space with $q_{0}=0$ predicts angular separations that are high by a factor of two at $z=2$. The assumption laden $\Lambda \mathrm{CDM}$ cosmology has also been plotted for comparison and is seen to lie even further from the data than the Friedman model.

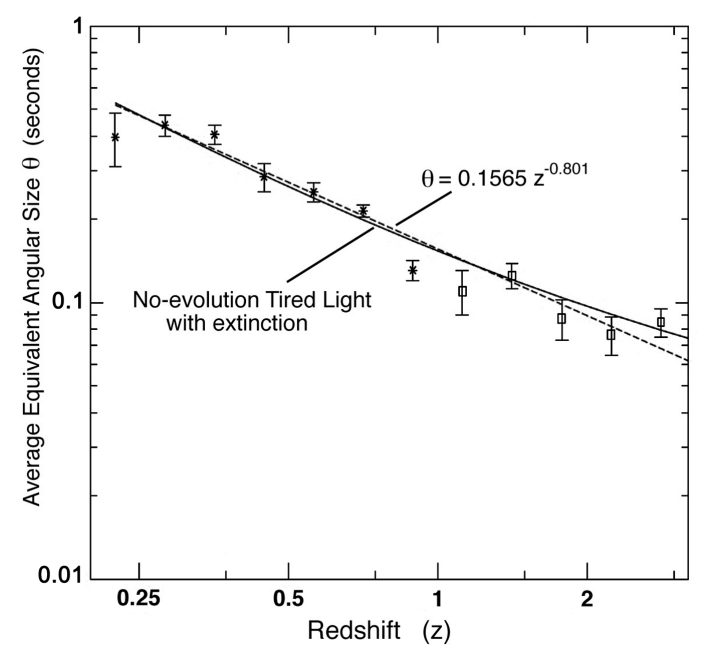

Figure 3. Comparison of the no-evolution tired light cosmology against data on the galaxy angular size-redshift test with the assumption of dust extinction of $a_{V}=3.4 \times 10^{-4}$ $\mathrm{Mpc}^{-1}$. 


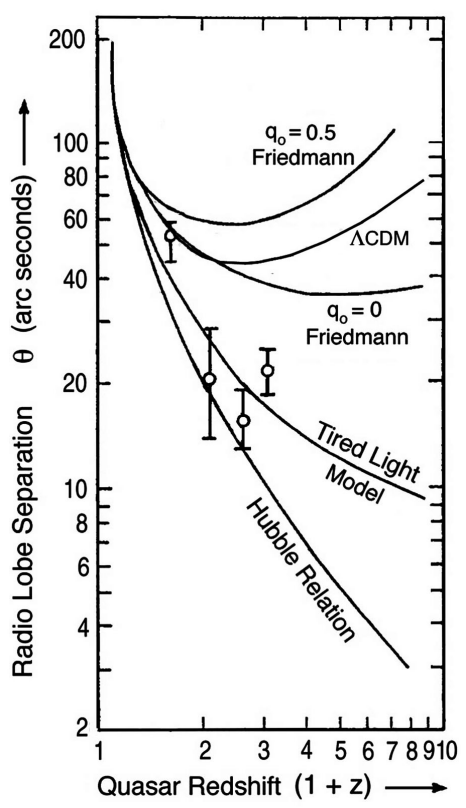

Figure 4. Median values of the angular separation of double radio lobes in quasars plotted against quasar redshift $1+z$. The angular data has been corrected for projection effects arising from the host galaxy's inclination to the line of sight. The tired light model is seen to make a far superior fit to the data in comparison to the various expanding universe predictions (after Ubachukwu and Onuora [9]).

For the Friedmann expanding universe model, or $\Lambda$ CDM cosmology, to attain a data fit similar to the tired light model, strong evolutionary effects would need to be introduced. That is, it would be necessary to invoke the ad hoc assumption that galaxy radio lobes had larger angular separations in earlier epochs and have been gradually decreasing in size over time. However, not only does this further increase the complexity of the expanding universe cosmology vis-a-vis the tired light cosmology, it also requires that one accept that galaxy cluster size and galactic radio lobe separation, which normally would not be expected to be related, both change over time in just the right manner so as to allow the expanding universe model to make a good fit to the data! One might indeed be justified in asking the question about the big bang hypothesis that cosmologist and radio astronomer K. Kellerman [8] posed almost 50 years ago, namely, "Are we drawing too many epicycles?" The law of parsimony would instead point to the tired light model as the candidate model that is capable of explaining the greatest amount of data with the fewest assumptions.

Hoyle [10] has noted that Friedmann models having $q_{0}>0$ predict a minimum angular size at some finite $z$, with the expectation that observed angular size should increase with increasing $z$. For example, a $q_{0}=0.5$ Friedmann model predicts a minimum radio lobe separation of around 1 arc minute, but no such minimum is observed in the data; see Figure 4. Sandage [11] has noted that this minimum has been sought for in many observational tests but has not been found. He points out that this failure is considered by cosmologists to raise serious doubts as to whether the cosmological redshift is indeed due to a real expansion of space. 
Many others have noted that the expanding universe hypothesis makes a poor fit against angular size redshift test data in both the radio, near infrared and visible parts of the spectrum. These include Kapahi [12], Andrews [13], Nabokov, et al. [14], and Lerner [15].

\subsection{The Galaxy Number Count Magnitude Test}

Another kind of cosmological test that has been used to check the predictions of cosmological models compares the differential galaxy number count, $\mathrm{d} N / \mathrm{d} m$ (the number of galaxies per square degree falling in a given apparent magnitude interval $\mathrm{d} m$ ), to the average magnitude of that interval, $m$. Figure 5 displays K-band data obtained up to the 25th magnitude that has been taken from Figure 1 of the paper by Totani, et al. [16]. This is compared to the no-evolution, tired light prediction (solid line), which is seen to make a relatively good fit to the data trend. The number counts for the tired light prediction were reduced in accordance with the selection effect correction which Totani et al. give in Figure 6 of their paper. The dot-dashed line branching above the tired light prediction represents the uncorrected tired light prediction. ${ }^{1}$

The expanding universe alternative predicts an additional dimming of galaxy apparent magnitude since it incorporates an additional factor of $(1+z)$ due to

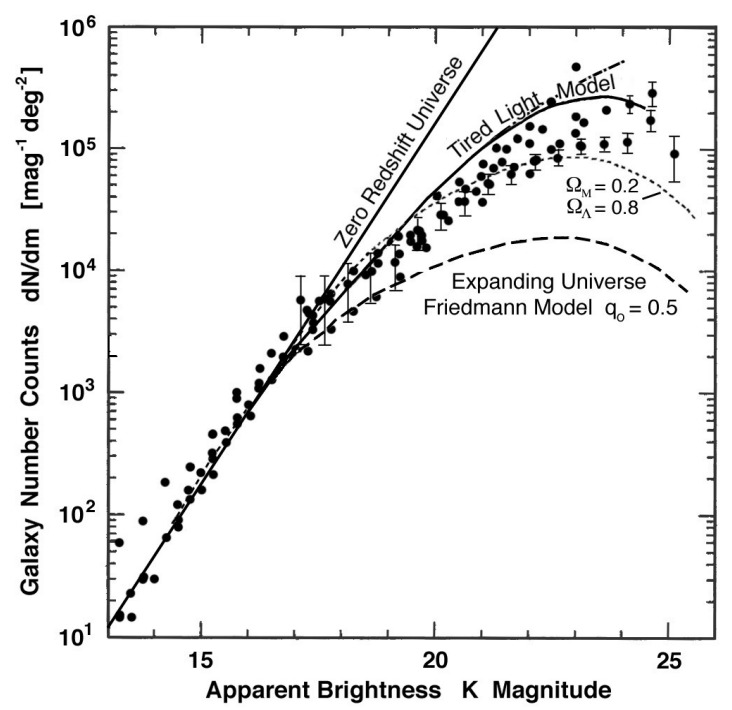

Figure 5. Differential galaxy number counts plotted against uncorrected galaxy K magnitude. Superimposed for comparison are the no-evolution, tired light model corrected for data selection effects (solid line), the tired light model with no correction (dot-dash line), the no-evolution, $\Omega(0.2,0.8)$ accelerating universe model with selection effect correction (dotted line), and the no-evolution, $q_{0}=0.5$ expanding universe model with selection effect correction (dashed line).

${ }^{1}$ The $\mathrm{d} N / \mathrm{d} m$ vs. $m$ dependence for a static, non-evolving Euclidean universe with no redshift dependent attenuation is represented by the sloping straight line in Figure 5 . In such a universe, the integral galaxy number count $N$ would increase with the cube of distance, $r$ as $N \propto r^{3}$. Galaxy brightness would decrease according to the inverse square of distance or expressed in terms of apparent magnitude $m$, it would vary as: $m \propto 5 \log (r / 10)$, or, $r \propto 10^{0.2 m}$. Combining these relations gives: $N \propto$ $10^{0.6 m}$, or similarly for differential counts: $\mathrm{d} N / \mathrm{d} m \propto 10^{0.6 m}$. 
the relativistic time dilation effect (the Hubble "number effect"). The $q_{0}=0.5$ no evolution, expanding universe prediction, which is plotted as the dashed line, falls substantially below the $\mathrm{d} N / \mathrm{d} m$ data trend on this test. The accelerating expanding universe cosmology modeled with $\Omega_{\mathrm{M}}=0.2$ and $\Omega_{\Lambda}=0.8$, plotted as the dotted line, fairs better, but is also assumption laden. This cosmology plot and the Friedmann cosmology plot are both taken from Figure 9 of Totani's paper and include corrections for data selection effects. The $q_{0}=0$ Friedmann model, not shown, would plot somewhat below the accelerating universe prediction. While the accelerating universe model fits the number count data better than the $q_{0}=0.5$ Friedmann model, it emerges as the less desirable alternative on the Tolman surface brightness test, considered in Section 2.3, since it requires a very large amount of luminosity evolution to secure a good fit on that test $(\approx 1.8 \mathrm{mag}$ nitudes at $z=0.9$ ).

The $q_{0}=0.5$ Friedmann model and the accelerating universe model of Totani et al. at $m_{K}>23$ can be made to fit the data only by introducing the ad hoc assumption that space was more densely populated with galaxies in earlier epochs and that galaxy number density has been rapidly decreasing over time. For the $q_{0}=0.5$ cosmology prediction, galaxies would have had to be 10 times more abundant at $m_{K}=22(z \approx 2.3, t \approx 10$ billion years lookback time) as they are at present. But then this raises the question whether it is justified to assume that the spatial population density of galaxies has been varying to such a great extent and in just the right manner so as to allow the expanding universe model to make a good fit to the data, given that the tired light cosmology already makes a reasonably good fit. In addition, LaViolette [2] has tested the tired light model on the radio galaxy differential number count test and finds that it makes a better fit than the expanding universe prediction. ${ }^{2}$

\subsection{The Tolman Surface Brightness Test}

The Tolman surface brightness test, devised in 1930 by Richard Tolman [18], provides another way of distinguishing the predictions of alternative cosmologies. This test uses galaxy surface brightness, $S$, as a distance indicator for comparison to galaxy redshift. The expanding universe model predicts that surface brightness should vary as the inverse fourth power of redshift, $S \propto(1+z)^{-4}$, one factor of $(1+z)$ being due to relativistic time dilation, one factor being due to ${ }^{2}$ Edward Wright [17] has contested LaViolette's conclusion that the no-evolution, tired-light cosmology makes a good fit on the radio galaxy differential number count test. His objection was that the tired-light cosmology does not fit the small dip in the data trend evident in number counts of the brightest sources, a data trend anomaly that comprises one hundredth of one percent of the total number of sources in that study. Kellermann and others, however, have questioned whether this minor number-count deficiency of bright sources is even real, suggesting that it may be an artifact of the poor sampling statistics in that part of the data set which samples nearby galaxies. By most standards, a model that fits 99.99 percent of a data trend would be considered to be a desirable choice, especially when compared with the non-evolving $q_{0}=0$ Friedmann cosmology which fits only a small fraction of the data points $\left(<10^{-4}\right)$. In 1989 LaViolette wrote to him pointing out that his refutation of the tired-light model is supported by very few data points. He unexpectedly responded that "a valid theory must fit all the data, not just 99.99 percent of it". To the contrary, given that number count data is inherently stochastic, it is meaningless to distinguish 99.99 percent from 100 percent. 
the cosmological (Doppler) redshift effect, and two factors being due to relativistic geometrical aberration [19]. The tired light model, on the other hand, predicts an inverse surface brightness-redshift relation of $S \propto(1+z)^{-1}$ involving just one factor of $(1+z)$, the decline in surface brightness being due exclusively to the photon energy loss that produces its nonDoppler redshift. Consequently, the tired light model predicts that galaxy surface brightnesses should appear far brighter than the expanding universe alternative.

In the present discussion, we consider a version of this test which Lubin and Sandage [20] [21] [22] [23] conducted in the optical part of the spectrum. Their test compared the expanding universe and tired light cosmology predictions to the average surface brightnesses of galaxies in three high-redshift galaxy clusters having redshifts of $0.76,0.9$, and 0.92 . In performing their cosmology test, they commit the same "sin" that many other cosmologists have done, which is to add evolutionary corrections to their data to allow their favored expanding universe model to make a good fit. Then once these corrections are added, they plot also the tired light model, noting that it lies further from their data set. On the basis of their single Tolman test, they then claim to have disproved the tired light model, as they state that their "Hubble Space Telescope data rule out the tired light model at a significance level of better than $10 \sigma$ ".

Lubin and Sandage had considerable flexibility in adjusting the expanding universe model to fit their data. On the one hand, they had a range of expanding universe cosmologies available to them, differing by the value of the deceleration parameter (e.g., $q_{0}=0,0.5$, or 1 ). On the other hand, for a given cosmology, they had a wide range of galaxy luminosity evolution models to choose from to close the gap between theory and observation. These assumed that galaxies were brighter in primordial times to varying extents, ranging from 0.6 up to 1.7 magnitudes brighter in the R-band at a redshift of $z=0.9$ [23]. From this wide range, they chose the $q_{0}=0.5$ cosmology as their best alternative and noted that closing its prediction gap required an assumed luminosity correction of $\Delta M(\mathrm{R})$ $=0.99 \mathrm{mag}$ for the R-band data for the $z=0.92$ cluster, and $\Delta M(\mathrm{I})=0.39 \mathrm{mag}$ and $0.44 \mathrm{mag}$ for the I-band data for clusters at redshifts $z=0.76$ and 0.9. But given the flexibility at their disposal in choosing a model to fit their data, is it not inappropriate for them to claim that they were checking the viability of the expanding universe cosmology relative to the tired light cosmology?

If their intention had been to make an equitable comparison, a better approach would have been to plot both cosmologies with no evolutionary corrections and compare each on multiple cosmology tests, a method advocated by LaViolette [2] and utilized as well in the present study. If they had, they would have seen that without evolution their favored $q_{0}=0.5$ expanding universe cosmology not only makes a poor fit to their data, but also makes a very poor fit on both the angular size redshift tests and galaxy number count test. If one refrains from making ad hoc evolutionary corrections and compares the alternative cosmologies to their R-band surface brightness data, it is found that the 
no-evolution, tired light cosmology in fact lies closer to the data trend than either of the two no-evolution, expanding universe cosmologies, the tired light model being $\approx 0.4$ magnitudes brighter than the data trend at $z=0.92$, while the $q_{0}=0.5$ and $q_{0}=0$ cosmologies are 1.2 magnitudes and 1 magnitude dimmer respectively. Here the tired light model predicts surface brightnesses that are slightly higher than the data trend, while the expanding universe cosmology predicts surface brightnesses that lie substantially below the data trend.

When the no-evolution cosmologies are compared to their I-band surface brightnesses data, the tired light prediction in this case lies further from the data trend, deviating by 0.89 magnitudes brighter at $z=0.90$, as compared with 0.45 and 0.65 magnitudes dimmer respectively for the $q_{0}=0.5$ and $q_{0}=0$ expanding universe cosmologies.

This comparison changes considerably if corrections are introduced for light extinction due to the presence of galactic and intergalactic dust, something that Lubin and Sandage did not do. Both Aguirre [24] [25] and Goobar et al. [26] argue that light extinction by intergalactic dust may be as high as 0.2 magnitudes at $Z=0.5$ in the case of an open universe cosmology with $\Omega_{\mathrm{M}}=0.2\left(q_{0}=0.1\right)$. According to Model B of the paper by Goobar et al. (Figure 9 and Figure 10 in their paper) galaxies at a redshift of $z=0.92$ would be dimmed by $\approx 0.33$ magnitudes in the $\mathrm{R}$ band and by $\approx 0.3$ magnitudes in the I band. Also, Rowan-Robinson [27] has proposed extinctions originating internal to the galaxy of 0.33 magnitudes for host galaxies in the redshift range $z=0.15$ to 0.8 . To correct the data for dimming due to both galactic and intergalactic dust extinction, it is here suggested that the R-band surface brightnesses which Lubin and Sandage report for galaxies in the $z=0.92$ cluster be increased by 0.4 magnitudes and the I-band surface brightnesses they found for galaxies in the $z=0.76$ and $z=0.9$ clusters be increased by 0.36 magnitudes, the dust extinction correction in the I-band being assumed to be slightly smaller.

With these extinction corrections, the data trends in the R-band data set would move upward by $0.4 \mathrm{mag}$, allowing the no-evolution tired light prediction to make a good fit, while increasing the discrepancy of their $q_{0}=0.5$ and $q_{0}$ $=0$ expanding universe predictions to $1.6 \mathrm{mag}$ and $1.4 \mathrm{mag}$ respectively. Even against their I-band data, which is about 0.4 to 0.5 magnitudes dimmer than their R-band data, the tired light cosmology with dust extinction makes a better fit than either expanding universe prediction. The tired-light model now would be 0.5 mag brighter than the data set, whereas the $q_{0}=0.5$ and $q_{0}=0$ cosmologies would now lie $0.8 \mathrm{mag}$ and $1.0 \mathrm{mag}$ dimmer than the data trend. To be fair, the tired light cosmology should be compared to the $q_{0}=0$ expanding universe cosmology since the $q_{0}=0.5$ cosmology requires the introduction of unsupported assumptions about the existence of hidden mass. So, even considering the I band cluster data of Lubin and Sandage, when dust extinction is assumed, the tired-light model makes a far closer fit.

It does not seem unreasonable to introduce the assumption of dust extinction since Lubin and Sandage used comparatively dim clusters in their study. For 
example, compared to the 102 clusters tabulated in the Hubble diagram study of Kristian, Sandage and Westphal [28] or the 119 clusters tabulated in the paper by Postman and Lauer [29], the three clusters that Lubin and Sandage use lie near the faint limit of the luminosity range in these other studies. In particular, the data points for the first ranked galaxies from each of the three high-z clusters they analyzed range from half a magnitude to a full magnitude dimmer than the data trend for the first-ranked galaxies in the data set of the Hubble diagram of Kristian-Sandage-Westphal (KSW) plotted in Figure 6 (Section 2.4).

To illustrate this the magnitudes of the first ranked galaxies in the three clusters studied by Lubin and Sandage are converted in Table 1 so that they may be properly compared with the KSW data. The magnitudes listed in columns (4) and (6) of Table 1 have been taken from Tables 5-7 of Lubin and Sandage [22] using their values for Petrosian galaxy radii of $\eta=1.7$, except for galaxy No. 9 ( $z$ $=0.76$ ) whose magnitude was available only for a Petrosian radius of $\eta=1.5$.

To convert the I-band magnitudes for the first two galaxies listed in the table to R-band magnitudes a Keck R-I color index correction is applied (col. 5). Also, a dust extinction correction similar to that applied to the Tolman test data is added (col. 7) to brighten the magnitudes of all galaxies. The magnitudes are then brightened by an additional -0.25 magnitudes (col. 8) since the photometry for the Kristian-Sandage-Westphal data are based on the Johnson-R system whereas that for the Lubin and Sandage data is based on the Cape-Cousins system, the latter magnitudes being fainter by a zero-point offset of 0.25 magnitudes; see footnote on p. 1072 in Lubin and Sandage [22]. When the corrected magnitudes (col. 9) are compared to the first ranked galaxies plotted in the KSW study, two are seen to be so dim as to lie to the far right of the plot in Figure 6, the third at $\mathrm{z}=0.9$ lying entirely off the right side of the graph.

In 2002, in correspondence with A. Sandage, the Author pointed out that the clusters they used in their study were relatively faint [30]. To this, Sandage replied [31]:

"... as you have noticed, the first ranked galaxy in each of the three clusters that we (Lubin/Sandage) have studied are fainter than the mean of the distribution of either the total sample of Postman and Lauer or of K/S/W. However, each of these brightest galaxies in our (Lubin/S) three clusters is still within the confines of the distribution of absolute magnitudes in either of these two lists $(\mathrm{P} / \mathrm{L}$ and $\mathrm{K} / \mathrm{S} / \mathrm{W})$, although, it is true, they are near the limit on the faint side. We have assumed that our three clusters are fainter than average in their brightest member, but not outside the known distribution for 'local' clusters."

So, in view of this admission, and the above magnitude comparison, the decision to brighten the Lubin-Sandage data set by the inclusion of a dust extinction correction appears justified and allows the tired-light model to make a superior fit to the Tolman test data. 
Table 1. Magnitude corrections applied to first ranked galaxies in the Lubin/Sandage data.

\begin{tabular}{ccccccccc}
\hline $\begin{array}{c}(1) \\
\text { Galaxy }\end{array}$ & Cluster & $z$ & $m_{I}$ & R-I & $m_{R}$ & $\begin{array}{c}\text { Dust } \\
\text { Correct. } \Delta m_{A}\end{array}$ & $\begin{array}{c}\text { K-correct. } \\
\Delta m_{K}\end{array}$ & $m$ corrected \\
No.9 & $1324+3011$ & 0.76 & 19.11 & 1.43 & 20.54 & -0.36 & -0.25 & $19.93 \pm 0.04$ \\
No.9 & $1604+4304$ & 0.90 & 19.99 & 1.61 & 21.60 & -0.36 & -0.25 & $20.99 \pm 0.09$ \\
No. 23 & $1604+4321$ & 0.92 & - & - & 20.39 & -0.40 & -0.25 & $19.74 \pm 0.08$ \\
\hline
\end{tabular}

In paper number IV of their Tolman test paper set, Lubin and Sandage [23] argue that standard luminosity evolution models require that galaxies should have been brighter in primordial times and that such a luminosity evolution assumption would move the tired light model prediction away from the data trend, instead of closer, to the model's detriment. However, their desire to apply the same luminosity evolution assumptions to the tired light model is poorly founded. For, static-universe tired light cosmologies require nonstandard models of stellar evolution. Indeed, when one adopts the tired light model as being the correct alternative, galaxy distances and look-back times increase in comparison with distances predicted by the expanding universe cosmology, which in turn affects the rate of galaxy evolution. Also as described below, static universe cosmologies lead in the direction of requiring a mechanism of continuous matter creation to explain the origin of matter, something that would drastically alter any assumptions about primordial galaxy evolution.

Lerner [32], Crawford [33], and López-Corredoira [34] have all been critical of the way Lubin and Sandage performed their study, and of their claim that their test data refutes the static universe, tired light model. Lerner [32] has performed a repeat Tolman test whose data set includes galaxies with redshifts of up to $z=6$ and concludes that the data is clearly compatible with the static universe hypothesis and clearly incompatible with the expanding hypothesis, even when reasonable brightness evolution is included. Also, Lerner, et al. [35] has performed a repeat of the Tolman test earlier conducted by Pahre, et al. [36] using UV surface brightnesses of galaxies having redshifts as high as $z \sim 5$. After correcting the errors made by its authors in their cosmology comparison, he demonstrated that the data in fact make a good fit to the static universe Euclidean tired light model and are incompatible with the concordance expanding universe cosmology. Andrews [37] has also compared the expanding universe model and the static universe, tired light model on a Tolman test and has concluded that the data conclusively favor a static universe over an expanding universe. Both of Lerner's test findings and those of Andrews are contrary to the conclusions of Lubin and Sandage.

\subsection{The Hubble Diagram Test}

The Hubble diagram test uses galaxy apparent magnitude, $m$, as a distance indicator for comparison to galaxy redshift. The $m-z$ curve for the no-evolution tired 
light prediction is given by the following equation:

$$
m=5 \log \left\{[\ln (1+z)](1+z)^{1 / 2}\right\}+C,
$$

where $C=19.8$ [38]. The first term, $\ln (1+z)$, results from the nonlinearity of the tired light redshift distance relation and is derived by substituting the tired light relation $r=\ln (1+z) / \beta$ into the magnitude relation $m=5 \log r+$ constant, with the $1 / \beta$ term being absorbed into the constant. The second term is the "energy effect", where galaxy dimming is due to the spontaneous diminution of photon energy with travel distance.

In the expanding universe cosmology, galaxy dimming is due both to the cosmological (Doppler) redshift effect and to relativistic time dilation, the latter effect causing the emitted stream of photons to spread out in time. It is also affected by the distances modeled for the galaxies, which differ from those in the tired light cosmology. The $m-Z$ curves that plot the standard no-evolution Friedmann model predictions are based on the equations of Mattig given as:

$$
\begin{gathered}
m=5 \log \left[z\left(1+\frac{z}{2}\right)\right]+C \quad\left(\text { for } \quad q_{0}=0\right) \\
m=5 \log \left\{2\left[1+z-(1+z)^{1 / 2}\right]\right\}+C \quad\left(\text { for } q_{0}=0.5\right),
\end{gathered}
$$

where $C=19.8$ [39]. At a given redshift, these expanding universe cosmologies predict magnitudes fainter than the tired light model.

Figure 6 plots the R-band magnitudes against redshift for the brightest galaxies in each of 103 clusters taken from Figure 4 of the 1978 paper of Kristian,

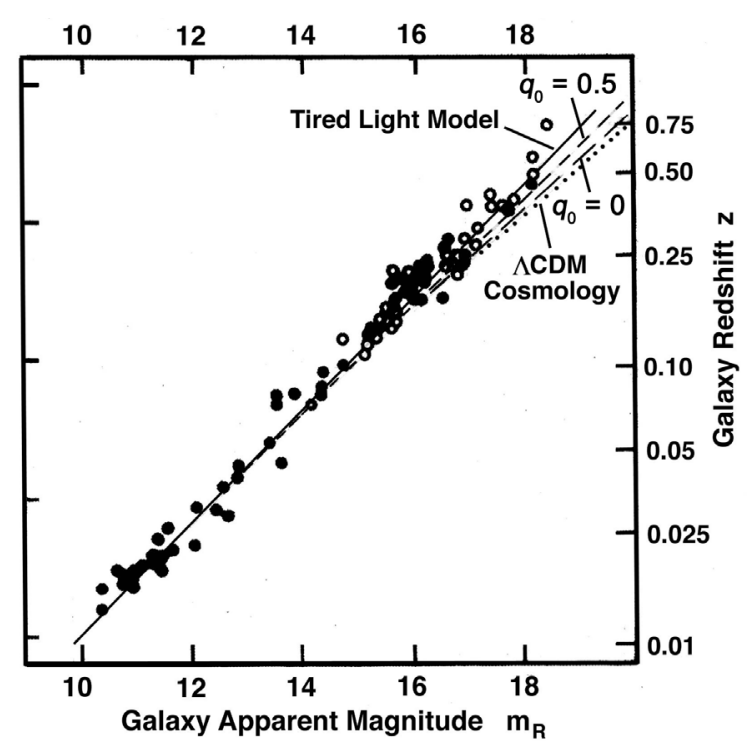

Figure 6. The Hubble diagram charts the R-band magnitude-redshift coordinates for the brightest galaxies in each of 103 clusters; data for $z<0.75$ is taken from Kristian, Sandage and Westphal (1978). The data is compared with the no-evolution tired light cosmology (solid line), the $q_{0}=0.5$, no-evolution expanding universe cosmology (short dashes), the $q_{0}=0$, no-evolution expanding universe cosmology (long dashes), and the $\Lambda \mathrm{CDM}$ cosmology (dotted line). 
Sandage and Westphal (KSW) [28]. Although the data is comparatively old and does not rise over $z=0.75$, it nevertheless is useful in this multi-test study as a means of constraining the predictions of competing cosmologies with respect to other tests. The data set is here compared to the no-evolution, tired light cosmology (solid line) the $q_{0}=0$ no-evolution Friedmann prediction (long dashed lines), the $q_{0}=0.5$ no-evolution Friedmann model (short dashed lines), and the $\Lambda \mathrm{CDM}$ cosmology (dotted line). The latter two cosmologies make less desirable comparisons since they have unsupported assumptions about the existence of hidden mass, dark matter, or dark energy.

So again, the tired-light model makes the better fit. Of the three expanding universe predictions, the $q_{0}=0.5$ Friedman model with the unsupported assumption of hidden mass comes closest to the data trend. However, choice of this cosmology over the $q_{0}=0$ cosmology worsens the fit of the expanding universe prediction on the angular size redshift tests, moving it further from the data trend on those tests. This shows the advantage of using multiple cosmology tests when one wishes to test competing cosmologies in a fair and consistent manner.

\subsection{The Photon Flight Time Redshift Test: A Version of the Hubble Diagram}

To make a more definitive judgment between expanding and static universe cosmologies using the Hubble diagram test alone, higher redshift data is needed. To this end, Marosi [40] has examined magnitude-redshift data for 280 supernovae and gamma ray bursts (GRBs) extending from $z=0.01$ to $z=8.1$ and finds that the tired light model makes a very good fit to the data over the entire data span. To better compare the competing cosmologies, rather than plotting redshift $z$ versus apparent magnitude, $m$, he plots redshift versus photon flight time, $t_{s}$. When this is done, the difference between the expanding and static universe alternatives becomes more apparent. The $t_{s}-z$ diagram shown in Figure 7

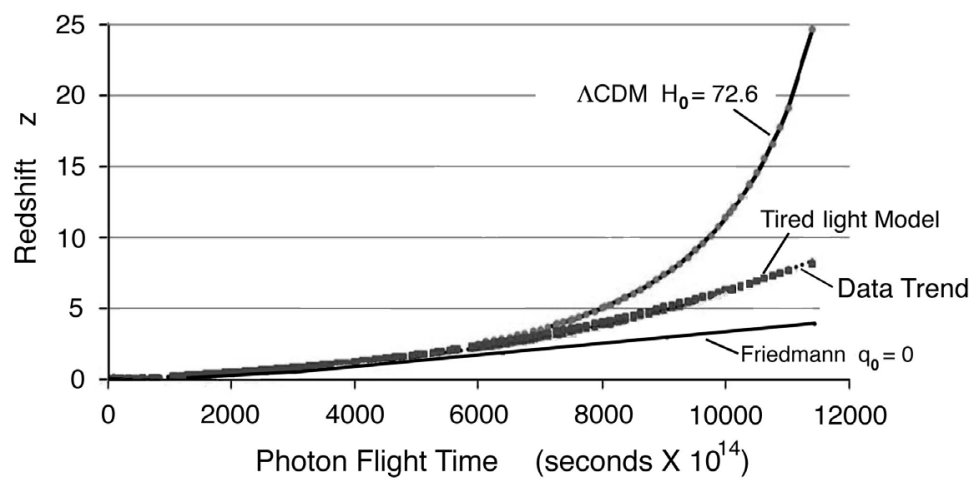

Figure 7. Redshift of type Ia supernova and GRBs as a function of photon flight time $t_{s}$. Plotted curves include the supernova data trend together with the tired light model having $H_{0}=2.024 \times 10^{-18} / \mathrm{s}$ (gradually curving line), and two expanding universe model predictions, the $\Lambda \mathrm{CDM}$ model with $H_{0}=72.6 \mathrm{~km} / \mathrm{s} / \mathrm{Mpc}$ (strongly curving solid line) and the $q_{0}=0$ Friedmann model (lower straight line). 
is adapted from Figure 3 of Marosi's paper. It plots the $\Lambda$ CDM cosmology $\left(\Omega_{\mathrm{M}}=\right.$ $0.266, \Omega_{\Lambda}=0.732, k=0$ ) with $H_{0}=72.6 \mathrm{~km} / \mathrm{s} / \mathrm{Mpc}$ (upward curving solid line after Marosi) and the exponential tired light relation the lower gradually bending line, which is virtually indistinguishable from the $t_{s}-z$ supernova/GRB data trend. The no-evolution $q_{0}=0$ Friedmann cosmology, the lower straight line, has been added to Marosi's plots for comparison. While this Friedmann model is currently not as popular as the $\Lambda \mathrm{CDM}$ cosmology, it is preferred in the present multi-test comparison since it makes no ad hoc assumptions about the presence of dark matter and dark energy.

The $\Lambda \mathrm{CDM}$ and Friedmann cosmologies both assume that the universe expands linearly according to $v=z c=H_{0} D_{c}$, where $D_{c}$ is comoving distance. $D_{c}$ values in Gly, obtained at various redshifts for the two expansion cosmologies, may then converted to $t_{s}$ values by dividing by $c=3.15 \times 10^{-17} \mathrm{Gly} / \mathrm{s}$. The no-evolution tired light model instead predicts an exponential relation between $t_{s}$ and $z$ of the form:

$$
\mathrm{e}^{H_{0} t_{s}}=1+z,
$$

Marosi found that when $H_{0}=2.024 \times 10^{-18} / \mathrm{s}$, this static universe cosmology yields a best fit to the data trend with a very high confidence level. Rewriting (11) as:

$$
H_{0} t_{s}=\ln (1+z)
$$

and substituting $t_{s}=r / c$ in the left term of Equation (12) yields essentially the $r$ - $Z$ relation of Equation (5).

As Marosi notes, the tired light prediction essentially closely overlays the supernovae $z-t_{s}$ data trend out to $z=8.1$, while the $\Lambda \mathrm{CDM}$ expanding universe cosmology with $H_{0}=72.6$ departs significantly from the data trend for redshifts $>3$. At $z=8.1$, this $\Lambda$ CDM cosmology predicts a value of $z \sim 25$, over 3 times greater than the data trend! Compared to the $\Lambda \mathrm{CDM}$ cosmology, $q_{0}=0$ Friedmann cosmology avoids making ad hoc assumptions about dark matter and dark energy. But it makes a poor fit for $z>2$, consistently predicting higher $\mathrm{t}_{\mathrm{s}}$ values than the data trend.

Marosi [41] has also performed a photon-flight-time vs. redshift test for 84 gamma ray bursts ranging from $z=0.033$ to $z=8.1$. The $t_{s}-z$ diagram shown in Figure 8 is adapted from Figure 4 of his 2019 paper. It plots the $t_{s}$ vs. $1+z$ data based on 84 statistically verified gamma ray burst redshift-magnitude data points together with the exponential prediction of the tired light model (line a), compared to the $\Lambda \mathrm{CDM}$ expanding universe model with $H_{0}=62.5 \mathrm{~km} / \mathrm{s} / \mathrm{Mpc}$ (line b), and $H_{0}=72.6 \mathrm{~km} / \mathrm{s} / \mathrm{Mpc}$ (line c). The $q_{0}=0$ no-evolution Friedmann cosmology is also added to Marosi's graph (line d). The tired light exponential relation is again virtually indistinguishable from the data trend whereas the expanding universe models noticeably deviate from the data trend. The $H_{0}=72.6$ $\Lambda \mathrm{CDM}$ model begins departing significantly from the data trend for $z>2$. The $H_{0}=62.5 \Lambda \mathrm{CDM}$ model overestimates the flight times for $z<6$ and underestimates the flight times for $z>6$. 


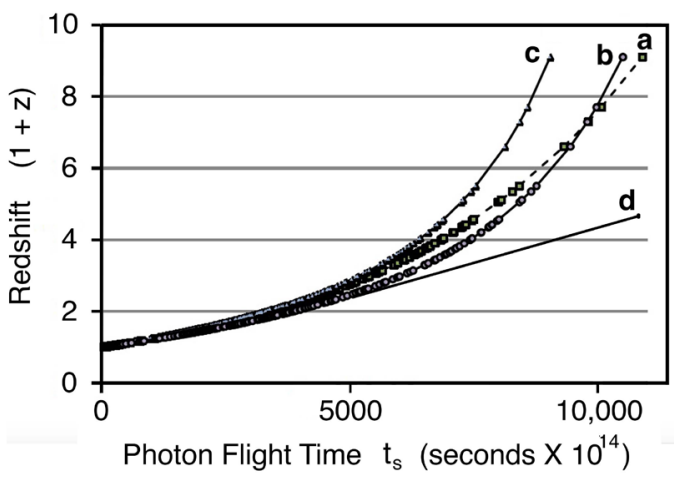

Figure 8. Redshift of gamma ray bursts as a function of photon flight time $t_{s}$. Plotted curves include the data trend and coincident tired light model prediction with $H_{0}=68.2$ $\mathrm{km} / \mathrm{s} / \mathrm{Mpc}\left(2.21 \times 10^{-18} / \mathrm{s}\right)$ (line a), the $H_{0}=62.5 \mathrm{~km} / \mathrm{s} / \mathrm{Mpc} \Lambda \mathrm{CDM}$ model (line b), the $H_{0}$ $=72.6 \mathrm{~km} / \mathrm{s} / \mathrm{Mpc} \Lambda \mathrm{CDM}$ model (line $\mathrm{c}$ ), and the $q_{0}=0$ Friedmann cosmology (line $\mathrm{d}$ ), here added to Marosi's graph.

Marosi finds that his data in Figure 7 is best fit with a Hubble value of $H_{0}=$ $2.024 \times 10^{-18} / \mathrm{s}$ and that his data in Figure 8 is best fit with a Hubble value of $H_{0}$ $=2.209 \times 10^{-18} / \mathrm{s}$. When expressed in velocity-distance terms, these yield Hubble constant predictions of $62.4 \mathrm{~km} / \mathrm{s} / \mathrm{Mpc}$ and $68.2 \mathrm{~km} / \mathrm{s} / \mathrm{Mpc}$. Averaged together, they predict a Hubble constant value of $H_{0}=65.3 \pm 2.9$. This tired light $H_{0}$ value lies about $1 \sigma$ above the $H_{0}=62.3 \pm 1.3 \mathrm{~km} / \mathrm{s} / \mathrm{Mpc}$ value determined by Sandage, et al. [42] using Cepheids in nearby galaxies to calibrate the distances and magnitudes of Type Ia supernovae in the redshift range $3000<v_{c m b}<20,000 \mathrm{~km} / \mathrm{s}$. It also falls somewhat above the $H_{0}=64.0 \pm 1.6 \mathrm{~km} / \mathrm{s} / \mathrm{Mpc}$ value determined by Tammann \& Reindl [43] using RR Lyr stars and stellar parallaxes to calibrate tip-of-the-red-giant-branch (TRGB) stars in halo population galaxies tied to Type Ia supernovae in the same redshift range used by Sandage, et al. The tired light determined $\mathrm{H}_{0}$ value lies somewhat below the $H_{0}=67.4 \pm 0.5 \mathrm{~km} / \mathrm{s} / \mathrm{Mpc}$ value derived from the $\Lambda \mathrm{CDM}$ model-dependent analysis of the CMBR reported by the Planck Collaboration [44]. It also falls substantially below the value $H_{0}=$ $69.8 \pm 0.8 \mathrm{~km} / \mathrm{s} / \mathrm{Mpc}$ obtained by Freedman, et al. [45] by calibrating TRGB in galaxies at distances of 7 to $20 \mathrm{Mpc}$ using eclipsing binary stars in the Large Magellenic Cloud and tying them to Type 1a supernovae. It also lies far below the $\Lambda \mathrm{CDM}$ cosmology value of $H_{0}=74.0 \pm 1.4 \mathrm{~km} / \mathrm{s} / \mathrm{Mpc}$ which Riess, et al. [46] have derived based on analysis of Cepheids in the Large Magellenic Cloud.

The discrepancy between these various $H_{0}$ determinations is found to be up to ten-fold greater than the accuracy of estimating the individual $H_{0}$ values. This conflict among determinations, termed the Hubble tension, has been problematic for the concordance cosmology. Some have suggested that the introduction of new physics may be needed to resolve it. Others such as Lombriser [47] and Ding, et al. [48] suggest that use of local calibrators may bias $H_{0}$ toward higher values since our Galaxy resides in an under dense region of the cosmos. Sandage, et al. [42] attribute these high $H_{0}$ values to a faulty choice of LMC P-L relations resulting in moduli that predict distances too short compared to their 
own. However, considering that the cosmology tests considered above collectively rule out the expansion hypothesis as the best choice cosmology, it seems more prudent to choose the $H_{0}=64.0 \pm 1.6$ value of Tammann and Reindl which approximates the $65.3 \pm 2.9 \mathrm{~km} / \mathrm{s} / \mathrm{Mpc}$ value that emerged from Marosi's tired light fit to supernovae and gamma ray burst data.

\section{Other Problems with the Expanding Universe Hypothesis}

\subsection{No Evidence for Time Dilation}

The expanding universe cosmology hypothesizes that high redshift galaxies are receding from us at close to the speed of light and that, due to the relativistic time dilation effect, clocks in those galaxies should be ticking slower or alternatively that supernova explosions should be taking longer to occur. One indication that we live in a cosmologically stationary, non-time-dilated universe comes from studies of gamma ray bursts. These are believed likely to be produced by supernova explosions [49]. So, if the light curves of distant supernova were in fact being time dilated, one would expect to see a similar effect in the duration of gamma ray bursts, the more distant, more highly redshifted gamma ray bursts being expected to last longer on the average. But such is not seen to be the case. It has now been established that X-ray bursts are essentially the same phenomenon as gamma ray bursts, except that they originate from much greater distances, the gamma rays in the original burst being cosmologically redshifted down to the X-ray energy band. However, the X-ray bursts are found to last about as long as gamma ray bursts.

Another study examined the durations of 195 Swift detected gamma ray bursts ranging out to a redshift of 8.1 yet found no evidence of time-dilation broadening in the light curves [50]. The data from this study, which is presented here in Figure 9, shows no evidence of any correlation between redshift and duration. Crawford [51] has also examined gamma ray burst data and finds no

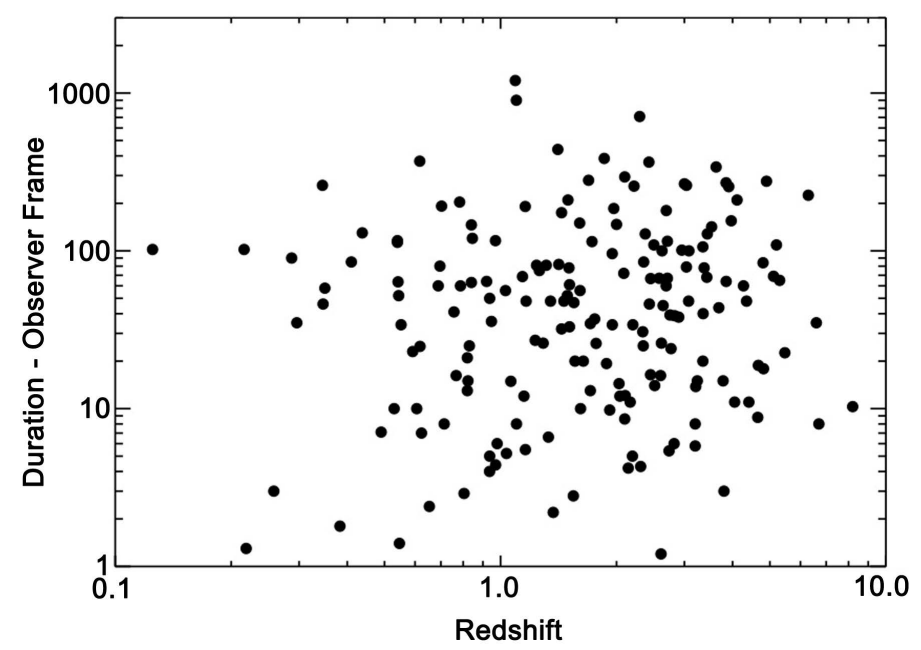

Figure 9. T90 duration vs. redshift for 195 Swift detected GRBs. Courtesy of D. Kocevski and V. Petrosian. 
evidence for any time dilation of their light curves. Quasar light curves also show no evidence of time-dilation broadening. One such study performed a Fourier analysis of the light curves of 800 quasars which were monitored on time scales from 50 days to 28 years to see if more distant quasars exhibited longer duration outbursts [52]. Low redshift quasars $(z<1)$ were compared to high redshift quasars $(z>1)$, but no evidence for time dilation was seen. The results of these various studies not only support the static universe, tired light cosmology, but also call into question the validity of the time dilation conclusions of the Supernova Cosmology Project (SCP).

Let us take a moment to analyze that study. The SCP study analyzed the durations of 60 supernovae out to a redshift of 0.83 and the authors claimed that their results indicated that supernova explosions in distant galaxies are time-dilated in accordance with the predictions of the expanding universe cosmology. They claimed their data was best fit by a $\Lambda \mathrm{CDM}$ cosmology having cosmological parameters $\Omega_{\mathrm{M}}=0.28$ and $\Omega_{\Lambda}=0.72$, and that their analysis refutes the static universe tired light model, which predicts that supernova duration should be independent of redshift. But the dependence of light-curve width on supernova redshift which their data showed was likely an artifact of data selection bias. In particular, this study is handicapped by the Malmquist bias, the tendency to preferentially detect intrinsically bright objects. As Phillips [53] has pointed out, searches for distant type Ia supernovae $(z>0.2)$ will clearly favor the discovery of superluminous events and this could introduce a significant Malmquist bias into the survey. At higher redshifts our telescopes necessarily sample a much greater volume of the universe and hence a much greater number of supernova-producing galaxies. In the redshift range 0.4 to 0.5 , one would be surveying a volume of space that was 34 times larger than that of the local neighborhood which spans the redshift range 0 to 0.1 . Going out to a redshift range of 0.7 to 0.8 , this observational volume increases to 66 times greater than the local volume. Hence because the high redshift domain subtends a very large volume of space, compared with the local environment, there is a much greater probability of observing extremely luminous high-energy supernovae, which normally occur very rarely. Since the light curve of such supernovae persist much longer than those of less luminous supernovae [54], high-z supernova searches will be skewed to discovering high-luminosity, long-duration supernovae, giving exactly the result that they found even with time dilation left out of the picture.

Moreover, shorter duration supernovae, being less luminous at maximum light, would not be as easily seen, especially at high redshifts where both the greater distances and greater dimming due to photon energy loss conspire to create conditions adverse to their detection. Hence there would be a tendency for supernovae with lower light curve width values to pass undetected resulting in a flux limited selection. Such flux limitation could explain why the SCP data set contains progressively fewer supernovae at progressively higher redshifts where instead progressively more supernovae should be observed due to the progressive increase in the volume of surveyable space. For example, the SCP 
data set contains 24 supernovae in the redshift range of 0.3 to $0.5 ; 13$ supernovae in the redshift range of 0.5 to 0.66 (defining a volume of space $30 \%$ larger); and only 3 supernovae in the redshift range of 0.66 to 0.85 (a $210 \%$ larger space volume). It is not a question of whether this data set might be flux limited. It is clear that it is flux limited since there is no other explanation that could account for this kind of number drop off. Standard theories of galaxy evolution cannot explain this drop off since such theories predict that high-z galaxies were bluer and had greater star formation rates with more frequent supernovae.

The occurrence in their data of a supernova at $z=0.46$ with a width factor of 2.26 provides evidence that some supernovae can have very long light curve durations, more than three times greater than what the time dilation assumption would predict. The width of this supernova even surpasses the widths of two supernovae that have almost twice the redshift. So, this extremely long duration event may simply be a supernova that is at the upper end of the spectrum in terms of luminosity and duration. Moreover, a decade after this SCP study was published, Quimby, et al. [55] reported discovering supernovae that are an order of magnitude brighter than type I supernovae and that take much longer to fade away. If we were to wait long enough, such long duration supernovae would be seen also in the local low-z neighborhood.

Although there is a tendency for brighter supernovae to last longer and to decline slower, supernovae having the same peak absolute magnitude can nevertheless have widely varying light curve decline rates, as represented by the parameter $\Delta m_{15}(\mathrm{~B})$, the amount that the supernova's B-band apparent magnitude decreases from supernova maximum by the 15th day. In Figure 7 of his paper, Rowan-Robinson [27] has plotted $\Delta m_{15}(\mathrm{~B})$ versus peak absolute magnitude for local supernovae that were first observed before their maximum and finds a large amount of data point scatter. For a given absolute magnitude, $\Delta m_{15}(\mathrm{~B}) \mathrm{du}$ ration can vary by up to $\pm 30 \%$. If we were to study a sample of supernovae collected over a much longer time period, say over two centuries, we would most likely find an even greater amount of data scatter perhaps as large as $\pm 50 \%$ since the more rarely occurring, brighter and longer lasting supernovae, would be more likely to be seen. So, to avoid a selection effect bias, a larger data scatter value of about $\pm 50 \%$ would be more appropriate when interpreting data observed at high redshifts. Given this amount of data scatter and a flux limitation inherent in observing at high redshifts, one is left to conclude that the high-z data published by Goldhaber et al. were significantly biased toward the high width factor end of the data scatter range and could have yielded width factors as much as $50 \%$ too high compared to the norm even with no time dilation effect.

A related problem with the SCP study is that the local neighborhood is under sampled. To get a fair sampling of supernova light curve widths in the local environment that would compare in a fair manner with what is seen at high redshifts, we would need to observe for a total of 260 years, or 65 times longer than the four-year period over which Goldhaber et al. collected their data. If the SCP group were to extend their study of the local environment for a few more centu- 
ries, quite likely they would discover supernovae that had durations just as long as the one's they observed at high redshift. Adding in these potentially observable long duration supernovae would shift the light curve width data upward toward higher width values.

In summary, due to the comparatively short time span for their search for local supernovae, the Supernova Cosmology Project's data set is biased toward charting lower width values at low redshifts. At the same time, due to flux limited sample selection effects their data set is biased toward charting higher width values at high redshifts. Together, these two effects conspire to produce the observed upward sloping trend line. With proper sampling, it is expected that such data would yield a flat trend line with no evidence for time dilation.

In Section 4 of their paper, Goldhaber et al. briefly acknowledge that their supernova data might suffer from such a selection effect bias. David Crawford [56] has also noted that the SCP supernova data set may be biased by selection effects that could affect the outcome of the study's time dilation conclusions. John Masreliez [57], another critic of the study's conclusions, also makes a convincing case that the SCP supernova sample is flux limited and that selection effects could entirely account for the $1+z$ rise in supernova light curve width factor. $\mathrm{He}$ also notes that the positive slope in the redshift-width relation arising from selection effects should have added on to the slope predicted by the $1+z$ time dilation effect, if in fact it were present, to produce a slope much steeper than 1. Since such a steep upward slope is not seen, we are left to suppose that there is no $1+z$ correlation and that the slope is due to other factors fortuitously mimicking the slope predicted by the expanding universe hypothesis.

As stated earlier, a particular cosmology cannot be proven or disproved on the basis of a single cosmology test. We must take a more holistic approach and view a cosmology's performance on several tests simultaneously. On the one hand, we have the supernova-light-curve-width test of Goldhaber et al. whose results are questionable due to selection effect biases and which favors a specific accelerating universe $\Lambda \mathrm{CDM}$ cosmology that does not perform well on other cosmology tests. On the other hand, we have the angular-size-redshift test, Tolman test, number count magnitude test, Hubble diagram test, and the photon-flight-time-redshift test, all of which favor the tired light cosmology which consistently makes a better fit to the data. To be considered remotely plausible the expanding universe hypothesis must be modified to include specific assumptions regarding the evolution of galaxy cluster size, galaxy radio lobe size, galaxy luminosity, and galaxy number density, etc. But the required assumptions are numerous, and some even produce opposing results, worsening the fit of the expansion cosmology on certain tests. The tired light model, on the other hand, being free from the need for ad hoc evolutionary corrections, is preferred on the basis of its simplicity.

\subsection{The Redshift Quantization Effect}

Tifft [58] [59] [60] [61] [62], Cocke [63], Cocke and Tifft [64], and Tifft and 
Cocke [65] find that cosmological redshifts are "quantized," in one sixth submultiples of $c \Delta z=72.45 \mathrm{~km} / \mathrm{s}$, i.e., $12 \mathrm{~km} / \mathrm{s}$, the $24 \mathrm{~km} / \mathrm{s}$ and $36 \mathrm{~km} / \mathrm{s}$ harmonics being most prevalent. After further study, Tifft [66] concluded that these other redshift quantizations were higher multiples of either $8.05 \mathrm{~km} / \mathrm{s}$ or $2.68 \mathrm{~km} / \mathrm{s}$ which he then regarded as the most basic quantizations (1/9 and 1/27 of the originally discovered unit). These findings have more recently been confirmed by Guthrie and Napier [67] and Napier and Guthrie [68] who find 37.5 and 71.5 $\mathrm{km} / \mathrm{s}$ periodicities in the redshifts of the local supercluster. The existence of the $72 \mathrm{~km} / \mathrm{s}$ periodicity is now well established with a probability of only $10^{-6}$ that it is due to chance.

Cocke and Tifft [64] suggest that the redshifts may be due to cosmological expansion and that the observed quantization indicates that the expansion of the universe is quantized, or that the universe is stationary and that the photon emission properties of atoms are quasi-stationary, for example, with the Rydberg constant monotonically changing its value over time in discrete steps. Here they may be alluding to the variable mass theory of Narlikar [69] which suggests that matter is being continuously created in the universe and that the inertial mass of matter gradually increases from the time of its creation, thereby creating a cosmological redshift-distance effect.

LaViolette [2] has proposed an alternative interpretation of this quantization effect suggesting that the observed incremental change of redshift represents discrete steps in the decay of photon energy as photons journey through space. Thus, rather than losing energy continuously, as Equation (1) describes, photon quanta would change their energy (and wavelength) in incremental fashion. Taking $\Delta r$ as the distance over which an average photon travels before undergoing a redshift transition of amount $\Delta z$, then over $\mathrm{n}$ increments it would accumulate a redshift of $n \Delta z$. Thus, given that $\Delta r=c \Delta z / H_{0}$, this implies $\Delta r=0.188$ Mpc, given that $c \Delta z \sim 12 \mathrm{~km} / \mathrm{s}$ and $H_{0}=64 \mathrm{~km} / \mathrm{s} / \mathrm{Mpc}$, if we adopt the value of Tammann \& Reindl [43]. So, a photon would travel a distance of about 610,000 light years before undergoing an incremental decline in energy and corresponding increase in redshift. Over extended distances tired light energy loss could be expressed as:

$$
Z=\mathrm{e}^{n \beta \Delta r}-1=\mathrm{e}^{n \Delta z}-1 .
$$

which is an updated version of Equation (4) above. On the other hand, if the expanding universe hypothesis were adhered to, it would be necessary to assume that space-time globally expands in quantized fashion, which appears to border on the incredulous.

Another interesting quantization result comes from the observations of Arp [70] which show that galaxy redshifts relative to the main galaxies in the Local Group, M31 and M81, and in the Sculptor Group are quantized in steps of approximately $72.4 \mathrm{~km} / \mathrm{s}$, matching the quantization interval that Tifft and Cocke find for more distant galaxies. Arp reports that since the redshifts are known with a precision of about $\pm 8 \mathrm{~km} / \mathrm{s}$, and for seven of these galaxies even more 
precisely, about $\pm 4 \mathrm{~km} / \mathrm{s}$, one is led to conclude that the galaxies in these groups are unusually "quiet" (relatively motionless). Observing that galaxy redshifts in the M31 and M81 groups are distributed around the $72.4 \mathrm{~km} / \mathrm{s}$ periodicity with a standard deviation of $17 \mathrm{~km} / \mathrm{s}$, Arp [71] has quite reasonably interpreted this as evidence that the peculiar motions of galaxies in these clusters can have velocities no larger than this. This presents a strong argument that the differential redshifts of galaxies in these local groups are not due to relative motion of the galaxies, but to some intrinsic photon energy change phenomenon.

\subsection{Multi-Megaparsec Structures}

Another problem with the expanding universe hypothesis is its inability to adequately account for the regular spacing of galaxy superclusters [72]. That is, as we look further and further out into space in the direction of the north and south galactic poles, the number of galaxies per unit volume is found to alternately increase and decrease in cyclic fashion. Galaxies group into wall-like structures that are seen to be spaced from one another by about $180 \mathrm{Mpc}$ along our line of site, their wave-like pattern being seen to stretch out $1500 \mathrm{Mpc}$ ( 5 billion l.y.) in either direction. This poses a problem for the expanding universe theory which predicts a space-time dimension doubling during that period. If the universe was expanding, and a supercluster wave pattern did for some reason emerge, its wavelength would be expected to vary with time, being twice as long now as it was 5 billion years ago. But this is not the case. The wavelength stays constant with look-back time. This is more logically explained if the universe is static and Euclidean and that galaxies in some manner became preferentially created in certain regions of space so as to form a wave pattern of cosmic proportions. Moreover, assuming that galaxy clusters had been initially uniformly distributed in space and typically had gravitationally induced peculiar velocities of $1000 \mathrm{~km} / \mathrm{s}$, the time taken to traverse $90 \mathrm{Mpc}$ to form just one of these supercluster aggregations calculates to be 100 billion years, which far exceeds the age of a big bang universe.

\subsection{The Age of the Universe}

Another difficulty with the concordance expanding universe theory is that it predicts an age for the universe that is too short in comparison to the ages found for the highest- $\mathrm{z}$ galaxies. For example, galaxies have recently been discovered having redshifts as high as 11.1 [73] and 11.9 [74]. According to the $\Lambda$ CDM $H_{0}=$ $70 \mathrm{~km} / \mathrm{s} / \mathrm{Mpc}$ concordance cosmology, a galaxy at $z=11.9$ would be seen in existence 300 million years after the time of the big bang. This would place it well before the reionization epoch which is theorized to have occurred around $z=7.7$ [44]. This raises the question of how this galaxy would have had time to develop given that recent models of galaxy formation indicate that it should take a galaxy at least 750 million years to form. How does cosmology explain that stars would have started to form and develop into a galaxy prior to the beginning of the big bang! For other problems with the big bang/expanding universe hypothesis, see 
López-Corredoira [34].

\section{Conservative and Nonconservative Tired Light Models}

It is useful to review here some history of the tired light model. A number of cosmologists have proposed energy conserving tired light mechanisms in which the energy lost from the original photon remains in the universe as low-grade heat. For example, seven months after Hubble published his redshift-distance relation, Zwicky [75], publishing in the same journal, proposed an energy-conserving tired light mechanism as a nonvelocity explanation of Hubble's findings. His theory assumed that photons have a nonzero rest mass and lose energy as a result of a gravitational drag resulting from their interaction with ambient matter. Alternatively, Pecker and Vigier [76] have suggested that cosmological photons have a nonzero rest mass and lose energy through their interaction with a bath of $\varphi$-particles that have masses much smaller than that of an electron. Also, Marmet [77] [78] [79] has proposed a conservative energy loss mechanism involving photon scattering from intergalactic hydrogen nuclei that has some basis in laboratory experiments. His experiments demonstrated that photon interactions with intergalactic gas should produce no angular deflection and hence no image blurring. Also, Zheng [80] has proposed that "soft photon" scattering from intergalactic electrons can cause redshifting. However, the scattering theories of both Marmet and Zheng have difficulty explaining why the cosmological redshifts are the same at radio frequencies since an intergalactic medium of hydrogen gas or electron ions would not scatter radio waves in the same manner as optical wavelength photons.

Image blurring and spectral flatness criticisms, however, do not apply to most non-conservative tired light mechanisms. James Clerk Maxwell may have been one of the first to propose nonconservative photon energy loss. His original electromagnetic wave equation contained the energy damping term $\sigma_{0} \mu_{0}(\partial \varphi / \partial t)$, where $\sigma_{0}$ and $\mu_{0}$ represented the electrical conductivity and magnetic permeability of background space [81] [82]. Nernst [83] [84] put forth a nonconservative tired light idea in which he proposed that Olber's paradox might be resolved if photons were assumed to undergo nonconservative energy damping during their journey through intergalactic space [85]. As in Maxwell's damped EM wave, in Nernst's version the lost energy was proposed to physically disappear from the universe. Vigier [86] has proposed a non-energy-conserving tired light model in which photons lose energy through energy dissipating interactions with stochastic vacuum fluctuations.

LaViolette [2] [87] has also proposed a non-conservative tired light effect. But the version he proposed was not devised specifically to explain redshift-distance observations, as was the case for the other tired light theories described above. Rather, his tired-light relation emerged as a prediction of the subquantum kinetics (SQK) physics methodology [87] [88] [89]. His main intention was to test the validity of this photon energy loss prediction against astronomical data. La- 
Violette's tired-light relation emerges from a methodology that adopts a very different approach to conceiving the nature of physical phenomena, nevertheless, is one that has been extensively published in the literature. It is advisable to summarize a bit about it here. According to SQK, all space is pervaded by a subquantum reaction-diffusion medium whose constituents are able to self-organize into concentration inhomogeneities thereby forming "bunched" field potentials at the quantum level that constitute observable subatomic particles and photons. The underlying reaction and diffusion processes of this medium are mathematically described by an open, nonlinear reaction system, termed Model G [87]-[93]. One characteristic of such systems is that the entropy or energy magnitude of a photon (reaction-diffusion wave) does not necessarily remain constant over time as is assumed in standard physics. Instead, its initial energy magnitude may progressively increase or decrease over time depending on the state of criticality of the underlying reactions.

Wave amplitude (energy) in such nonlinear open reaction systems is generally described by the following wave equation which is applicable to reaction-diffusion waves consisting of small amplitude excursions $[\varphi]$ from the ambient potential [94]:

$$
[\varphi]=\left[\mathrm{A}_{0}\right] \mathrm{e}^{i\left(\kappa_{R} r-\omega t\right)} \mathrm{e}^{-\kappa_{i} r},
$$

where $\mathrm{A}_{0}$ is the initial magnitude of the wave (photon) and where $\kappa_{R}$ and $\kappa_{i}$ are the real and imaginary parts of its wave number $\kappa$. The frequency and wavelength of the wave are given respectively as $f=\omega / 2 \pi$ and $\lambda=2 \pi / \kappa$ and the wave velocity is given as $c_{0}=f \lambda=\omega / \kappa$. The oscillatory real term in Equation (14), the first exponent on the right, is consistent with energy wave behavior in standard physics. The imaginary term, the second exponent on the right, though, is new to quantum electrodynamics/physics. It dictates nonconservative wave damping when $\kappa_{i}>0$ (when subcritical conditions prevail in the reaction system) and nonconservative wave amplification when $\kappa_{i}<0$ (when supercritical conditions prevail in the reaction system).

Equation (14) may be restated as follows to portray the manner in which photon energy changes as a function of photon travel distance in SQK:

$$
E(r)=E_{0} \mathrm{e}^{-\left(\alpha \varphi_{g} / c\right) r} .
$$

$E(r)$ signifies the wave's electric potential amplitude, or energy, at distance $r$, and is equivalent to $[\varphi]$ in Equation (14). Term $E_{0}$ represents the wave's initial electric potential amplitude, or energy, at $r=0$ and is equivalent to the wave amplitude term $\left[\mathrm{A}_{0}\right]$ in Equation (14). The exponent $\mathrm{e}^{-\left(\alpha \varphi_{g} / c\right) r}$ is essentially the same as the second exponent in Equation (14) where $\kappa_{i}=\alpha \varphi / c$. Here, $\alpha$ is a constant of proportionality, $c$ is the velocity of light, and $\varphi_{g}$ signifies the ambient gravity potential in the wave's vicinity. This serves as the bifurcation parameter determining the system's mode of behavior. Perfect energy conservation holds when the photon is traveling through regions of space sufficiently close to galaxies where the gravity potential is at its critical threshold, zero value, $\varphi_{g}(r)=0$. In the im- 
mediate vicinity of galaxies and galaxy clusters, where $\varphi_{g}(r)$ is negative, the subquantum reaction-diffusion processes become supercritical and dictate photon energy amplification. In intergalactic space, where $\varphi_{g}(r)$ becomes positive, the underlying reaction-diffusion processes become subcritical and dictate photon energy damping. These various modes of photon behavior are illustrated in Figure 10. This ability for gravity potential to affect the nature of photon energy conservation according to the gravity theory of SQK, of course, is not predicted by general relativity. Nevertheless, the gravity theory of SQK does predict effects consistent with all other aspects of general relativity [91] [95].

Since a photon from a distant galaxy would spend far more time traveling through subcritical intergalactic void regions than through supercritical regions surrounding galaxy clusters, its energy on average would progressively decline, in accordance with Equation (15). The average rate of energy attenuation that a photon would experience during its flight may then be expressed as:

$$
E(r)=E_{0} \mathrm{e}^{-\beta r} .
$$

where $\beta$, the average attenuation coefficient takes the place of term $\alpha \varphi_{g} / c$ in Equation (15). Expressed in terms of photon wavelength, $\lambda$, this would be rewritten as:

$$
\lambda(r)=\lambda_{0} \mathrm{e}^{\beta r} .
$$

This is essentially the same as the "tired light" relation which historically has been devised to explain cosmological observations. Or, if photon energy loss and redshift occur in quantum increments, as portrayed earlier by Equation (13), Equation (17) could be written as:

$$
\lambda(r)=\lambda_{0} \mathrm{e}^{n \beta \Delta r} .
$$

This SQK cosmological redshift relation was not observationally motivated to explain cosmological data, as had been the case for other tired-light models, but rather emerged as a corollary of the Model $\mathrm{G}$ reaction-diffusion system. The value of $\beta$ given above is chosen to be $\beta=H_{0} / c$, where $H_{0}$ is the observed value for the Hubble constant. Here we may choose the value $H_{0}=64.0 \pm 1.6 \mathrm{~km} / \mathrm{s} / \mathrm{Mpc}$,

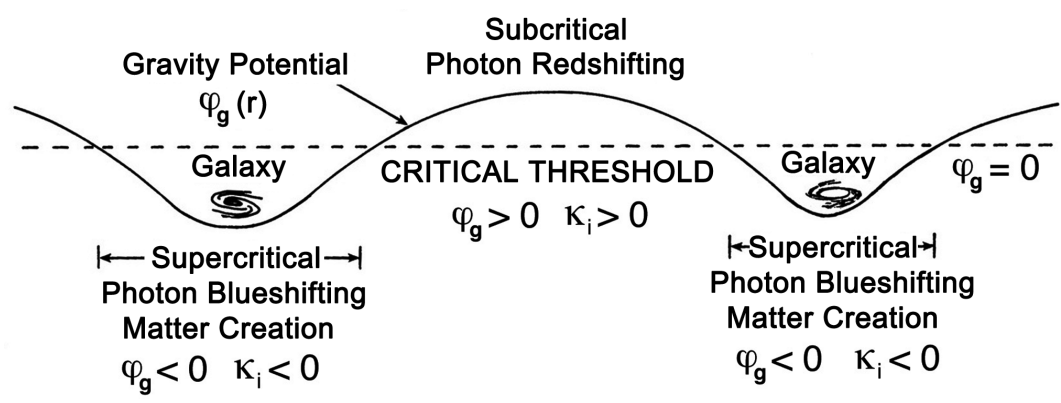

Figure 10. According to SQK, a photon's energy behavior depends on the ambient value of the gravity potential in the photon's vicinity judged relative to the critical threshold value, $\varphi_{g}=0$. Photons are predicted to progressively increase their energy within the supercritical gravity wells that surround galaxies and galaxy clusters (where $\varphi_{g}(r)<0$ ), and to progressively decrease their energy in intergalactic space (where $\varphi_{g}(r)>0$ ). 
as proposed by Tammann and Reindl [43], which falls close to the $H_{0}$ value indicated by the fit of the tired light relation on the $t_{s}-z$ cosmology tests of Marosi discussed above. This yields a photon energy decline rate of $\beta=6.54 \pm 0.16 \%$ per billion light years (bly). Expressing $\beta$ in time units, by multiplying by $c=3.17$ $\times 10^{-17} \mathrm{bly} / \mathrm{s}$, yields an energy loss rate $\mu=-\beta c=-2.07 \times 10^{-18} / \mathrm{s}$. This is about 10 orders of magnitude smaller than the smallest change observable in the laboratory. Hence such a nonconservative energy loss certainly has no observable influence on laboratory measurements. It becomes important only at the astronomical scale, and as has been seen above, it makes a good fit to astronomical data.

The cosmology test results discussed in Section 2, which vindicate the no-evolution, static universe tired light cosmology, are generally valid for all tired light models discussed above. However, the SQK tired light relation has a number of advantages over other tired light theories that have been proposed. First, since it predicts that the energy of the photon decays over time without emitting secondary photons from recoils with intergalactic particles, the photon should suffer no angular deflection that might contribute to image blurring. ${ }^{3}$ Second, by assuming that Model G operates sufficiently close to its threshold of marginal stability, its tired light energy loss effect will show no detectable frequency dependence. Hence radio frequency photons should redshift at the same rate as visible photons [91].

Third, the SQK paradigm, predicts that as stellar gravity fields extend outward away from their parent galaxy, beyond $\sim 3 \mathrm{kpc}$ from their source masses, their potentials decline and ultimately approach the steady state zero-gradient potential value present in intergalactic space. This is illustrated in Figure 10 by the ambient value attained in the subcritical space between the two galaxies. Hence over great distances the force of gravity departs from a Newtonian radial inverse square decline, as is the case in Milgrom's theory of modified Newtonian dynamics (MOND). As a result, in SQK, there is no gravitational potential summation problem leading to universal gravitational collapse, a difficulty that plagues many static universe cosmologies. Like the tired light effect, this limited range for gravity emerges as a prediction of SQK Model G and is not introduced as an ad hoc assumption [91].

A fourth advantage of the SQK tired light model is that its energy attenuation coefficient can vary according to the value of the ambient gravity potential. Thus, photons crossing subcritical void regions where the ambient gravity potential is substantially positive would exhibit a Hubble constant value much greater than that characterizing their passage through more typical regions of intergalactic space where their trajectory would occasionally traverse supercritical gravity well regions associated with clusters and superclusters. Such a gravity ${ }^{3}$ In their Tolman test cosmology paper, Lubin and Sandage [23] incorrectly suggested that the tired light mechanism that LaViolette [2] described in his 1986 paper accomplished its photon energy loss through photon scattering and commented that such scattering would inappropriately cause the images of distant galaxies to blur. This was an apparent misreading of his paper since LaViolette instead proposed a spontaneous nonconservative energy loss mechanism for the cosmological redshift. 
potential dependency could explain why voids in redshift space are found to appear elliptical with their direction of elongation oriented along the line of sight to observer, this being the so-called Fingers-of-God effect. That is, if photons undergo a greater than normal redshift rate as they travel through a void, galaxies on the void's far side would appear to have a greater than normal redshift and therefore be displaced away from the observer in redshift space, creating an apparent elongation [91] [95] [96]. Alternatively, SQK predicts that blueshifting would occur when photons pass through galactic clusters or superclusters, an effect that is able to account for the Kaiser effect and for the Fingers-of-God effect seen there as well [91] [95] [96].

A fifth advantage of the SQK tired light model is that the Model G reaction system from which it is derived also predicts that matter should be continuously created in supercritical regions of space. Consequently, with the emergence of the static universe tired light model in cosmology, SQK has a matter creation theory ready to explain the origin of the universe in lieu of the big bang explanation; see next section.

\section{A Theory of Continuous Creation}

The cosmological test data results summarized above lead inevitably to the conclusion that the universe must be globally static, and that the cosmological redshift is due to a process other than recessional velocity. We find then that the big bang theory is no longer a viable theory to account for the origin of things and that we must look elsewhere for alternatives to explain the nucleation of matter and energy quanta, a cosmology that does not require creation to take place all at once in a singular primordial space-time explosive event. One is then left to consider the possibility that matter might be continuously created throughout a cosmologically static universe. In some ways this scenario is more plausible than a big bang. For instead of being asked to believe that all the matter-energy of the universe was born into existence in a brief instant, we may now consider individual subatomic particles springing into being in a leisurely manner throughout the vastness of space at a rate so slow as to be virtually undetectable in the laboratory.

The idea that matter might be continuously created in regions of high matter density dates back to Sir James Jeans [97] whose observations of galaxies led him to speculate that there is a progressive evolution of galactic morphology from elliptical to spiral which involves a centrifugal ejection of material from their centers. Some years later he proposed that matter was being continuously created throughout the universe, at a time when the universe was believed to be cosmologically static. He held that the centers of galaxies may be similar to singular points at which matter pours into our universe from some other external dimension [98]. In his scheme, matter creation occurs in a nonconservative manner, as he essentially proposes that our universe may behave as an open system, at least in the cores of galaxies which he believed to be the most active creation centers.

Jean's idea of galaxy evolution was supported by Hubble [99] who found that 
for galaxies of a fixed total magnitude, the major axis diameter of a galaxy progressively increases as one proceeds through the morphology sequence from E0 to Sc. He embodied this sequence in his well-known "tuning fork-like" galaxy classification scheme. This implied to him that the size and mass of a galaxy progressively increases as one proceeds from early to late type galaxies. He wrote that the entire series can be represented by various configurations of an originally globular stellar mass expanding equatorially.

William McCrea [100] was also led to the idea of continuous matter creation on the basis of astronomical observation. He proposed that matter is continuously created throughout space, with creation being assumed to proceed most rapidly in regions of negative gravitational field potential, e.g., within stars and condensed masses. He proposed that all matter may potentially promote the creation of new matter. Furthermore, he proposed that since all matter normally resides in galaxies, the creation of fresh matter promotes the growth of galaxies. He notes that when on occasion a clump of matter becomes detached from its galaxy, it may serve as the embryo for a new galaxy.

Victor Ambartsumian [101] [102] further developed Jeans' idea proposing that a galaxy's evolution was largely shaped by energetic events taking place in its nucleus. Based on his observations of a variety of galaxies, he concluded that supermassive cores, most evident within the more massive galaxies, not only emit tremendous amounts of energy during their active phase, but large quantities of matter as well. He proposed that matter is thrown off in the form of an intense flux of relativistic particles as well as in the form of nonrelativistic ejections. He suggested that the latter would include gas moving at speeds of thousands of $\mathrm{km} / \mathrm{s}$, eruptive ejections of entire gas clouds, and even ejections of entire "galaxy embryos", i.e., supermassive cores. J. L. Sérsic [103], who echoed the ideas of Ambartsumian, suggested that giant elliptical galaxies with active cores may on occasion explosively fragment to produce progeny galaxies which could develop into various morphologies. Halton Arp [104] [105] also espoused similar ideas. Based on his observations of active galaxies, he concluded that active galactic nuclei can fission and explosively eject highly energetic clumps of matter, usually at steep angles to the galactic plane.

The novel physics methodology of SQK predicts a nonconservative matter creation cosmology that is consistent with the proposal of McCrea, as well as those of Jeans, Hubble, Ambartsumian, Sérsic, and Arp. Besides predicting tired light energy loss for photons traveling through intergalactic space, the Model G open reaction-diffusion system also predicts that matter may be spontaneously created in supercritical regions of space, this occurring wherever the ambient gravity potential lay slightly below its critical threshold zero value [89] [91] [106]. Such matter-spawning supercritical regions would prevail over large stretches of space scattered throughout the cosmos. In such regions, a zero-point energy fluctuation of sufficient magnitude is able to nucleate the formation of a neutron. Just as the energy of a photon would continuously blueshift in a supercritical environment, so too a zero-point energy fluctuation of critical size, sto- 
chastically arising in a matter-free region of space, would be able to grow in size. Rather than growing indefinitely, its magnitude instead converges to a new stable state, locally changing the former homogeneous steady-state of the reaction-diffusion system, the vacuum state, to an inhomogeneous steady-state as it forms a stable subatomic particle, i.e., a dissipative soliton wave pattern of well-defined wavelength and field potential magnitude. In SQK, this matter creation process is termed parthenogenesis, meaning virgin birth.

This parthenogenic matter creation process is illustrated in computer simulations of the partial differential equation system that constitutes Model G. A simulation showing the successive growth of an initial electric potential seed fluctuation into a dissipative soliton field pattern representative of a neutron is shown in Figure 4 of Pulver and LaViolette [93]. Also, a video simulation showing this particle materialization process may be viewed at: https://tinyurl.com/ybfphshf. As LaViolette [90] has shown, the emergent neutron has a form closely resembling that observed for the neutron in particle scattering experiments conducted by Kelly [107]. That is, the electric charge density distribution of the neutron's core is found to have a haystack-like shape and be surrounded by a radial periodicity. In fact, the electric potential field pattern for the Model $\mathrm{G}$ neutron yields a closer resemblance to Kelly's results than any previous nuclear field model [90].

To nucleate a neutron, the seed fluctuation must be of positive charge since in Model G positive fluctuations generate supercritical gravity wells which allow the fluctuation to grow and ultimately spawn a fully grown particle. Once formed, the particle's $\varphi_{g}(r)$ well continues to stabilize the particle, allowing the particle to persist in spite of potentially destabilizing zero-point energy fluctuations arising in its an environment. Seed fluctuations of negative potential, which might otherwise spawn the antiparticle state, e.g., the antineutron, fail to grow spontaneously since they generate a subcritical gravity potential hill in their vicinity and hence are self-extinguishing. Because of this matter-antimatter bias, the SQK matter creation process leads to a universe filled mainly with matter, rather than antimatter. This is an advantage since to date there has been no detection of antimatter galaxies. The apparent lack of an equal amount of antimatter in the universe has been a major setback for the big bang theory.

Besides creating a supercritical gravity well in its core, the SQK neutron also produces a peripheral gravity well shell at one Compton wavelength intervals from its center. The inner most two gravity well shells serve as supercritical regions where a randomly emerging zero-point energy fluctuation can self-amplify and nucleate a daughter neutron. Computer simulations of Model G have borne out this mother-daughter matter creation process; see Pulver and LaViolette [93]. Also, a video simulation showing this mother-daughter materialization process may be viewed at: https://tinryurl.com/yde4bvk3.

Once a neutron self-nucleates there is a very high probability that it will decay into a proton, beta particle, and neutrino before it has a chance to spawn a progeny particle. So, protons being stable particles would tend to accumulate in space as the most common matter nucleation sites. According to SQK, the pro- 
ton has a periodic Turing wave pattern very similar to that of a neutron, except that its core electric field would be positively biased relative to that of a neutron. Such biasing of the proton's wave pattern was also borne out by the particle scattering results of Kelly [107] which reveal that the proton's charge density profile is in fact positively biased relative to that of the neutron.

The probability of matter creation occurring in the immediate vicinity of an existing nucleon greatly outstrips that of a particle nucleating on its own in empty space. Consequently, with Model G this mother-daughter creation process becomes the dominant means of matter creation, with matter producing more matter at an exponentially increasing rate. Although initially devised for the purpose of modeling subatomic particles, Model G leads to a continuous creation scenario that is generally compatible with that of McCrea [100] wherein matter is continuously created throughout space with the creation process proceeding most rapidly in regions of negative gravitational field potential.

Nascent particles would first emerge as neutrons, whether they did so autonomously or assisted by the supercritical region of an existing nucleon. Neutrons emerging in the vicinity of a proton could either detach to continue as isolated neutrons that would later decay or could form a nuclear bond with its proton parent transforming it into a deuteron. Alternatively, a deuteron could serve as a nucleation site for a nascent neutron which could either detach from its parent or form a nuclear bond, transforming the deuteron into a tritium particle. This nuclear transmutation sequence could conceivably repeat causing a tritium nucleus to transform into ${ }^{3} \mathrm{He}$, and finally into ${ }^{4} \mathrm{He}$. Further transformation would be unable to occur through parthenogenic creation. Admittedly, the synthesis of $\mathrm{D},{ }^{3} \mathrm{He}$, and ${ }^{4} \mathrm{He}$ would be rare events. The main production of these elements, including $\mathrm{Li}$ and the light elements would occur later through thermonuclear fusion once stars had formed and grown sufficiently massive. Burbidge and Hoyle [108] have shown that stellar fusion could account for the observed abundances of these elements and that big bang nucleosynthesis is unnecessary.

Beta decay protons and electrons would be the most common particles existing in primordial times hence producing a diffuse ionized hydrogen gas heated to an X-ray emitting plasma state by collisions with the $0.78 \mathrm{Mev}$ beta particles. This could account for the observed diffuse X-ray emission that is observed coming from all directions of space. This X-ray emitting intergalactic gas has been referred to as the Warm Hot Intergalactic Medium, or WHIM. Its presence is also indicated by the so-called Lyman alpha forest, diffuse Lyman alpha emission radiated by the ionized portion of this gas. The big bang theory cannot account for its temperature because it predicts that the gas of its initially hot fireball should have long ago cooled down. Crawford [33] [109] has shown that the electrons in such a heated X-ray emitting plasma have a temperature and density sufficient to generate the observed $2.73^{\circ} \mathrm{K}$ cosmic microwave background radiation (CMBR). The source of ionizing radiation for the WHIM has puzzled astronomers since no stars are visible in these clouds and radiation from active ga- 
lactic cores falls short of the energy requirements. LaViolette [91], however, has shown that beta decay of parthenogenic neutrons would supply more than enough energy to power this emission. Moreover Arp, et al. [110] have argued that iron whiskers present in intergalactic space could thermalize the $3^{\circ} \mathrm{K}$ radiation field while allowing transparency at other wavelengths. So, with the theory that the WHIM may be the source of the CMBR we still retain the idea that the microwave background is of cosmic origin, energized not from a big bang, but from beta particle radiation arising from the decay of continuously created neutrons. Although, Burbidge and Hoyle [108] have also suggested that hydrogen burning in stars may be an energy source contributing to the $3^{\circ} \mathrm{K}$ radiation.

There is no 13.8 to15 billion-year time restriction in which to generate the cosmos in the SQK continuous matter creation cosmology. Once some regions in the WHIM had cooled sufficiently to allow hydrogen to condense into its liquid state, hydrogen could eventually coalesce to form comet sized bodies. Such planetesimals would grow both through accretion and through internal matter creation. As time passed, the planetesimal would grow in size, becoming a brown dwarf, then a red dwarf, and then a sun-like star. In the early stages of matter creation, isolated particles would have been the predominant sites of matter creation. Later, once matter had condensed to form material bodies and stars, the matter creation rates per nucleon would have risen considerably since the gravity potential well for such bodies would be far deeper than that of an isolate baryon. Such bodies would be the seat of both matter creation as well as energy creation through photon blueshifting.

The rate at which neutrons would self-create in the vicinity of nucleons located inside a star would depend not only on the ambient gravity potential within the star, which in turn determines the prevailing degree of supercriticality, but also on the star's internal temperature. Temperature would be a factor since thermal collisions would induce field potential fluctuations in the star's nucleons which would help to excite the materialization of neutrons. In general, the rate of matter creation per nucleon within a stellar body would far outstrip that occurring in space. Equation (19) gives a relation, admittedly tentative, of how a stellar body's rate of matter creation ( $\mathrm{g} / \mathrm{s})$ may be modeled:

$$
\frac{M}{d t}=k_{g} \cdot \bar{\varphi}_{g} \cdot M \cdot \bar{T}^{1 / 2},
$$

$k_{g}=10^{-40} \mathrm{~s} / \mathrm{cm}^{2} / \mathrm{K}$ being a constant of proportionality, $\bar{\varphi}_{g}\left(\mathrm{~cm}^{2} / \mathrm{s}^{2}\right)$ being the body's average internal gravity potential, $M$ (grams) being the body's mass, and $\bar{T}$ (K) being its average internal temperature [91] [106].

As the star's core grew past a critical temperature and density, nucleosynthesis would commence. The gas expelled in the star's stellar wind would generate a surrounding nebula in which orbiting gaseous planets would form. Over time these daughter planets would themselves grow into stars. Meanwhile the primordial mother star would continue to grow, proceeding up the stellar main sequence until it became a blue supergiant. This would either explode as a super- 
nova or discharge its atmosphere leaving behind a white dwarf, bare stellar core. This core would not be a dead star energized only by matter accretion, as standard physics proposes, but would be a stellar body that would continue to grow by internal matter creation. Hydrogen continuously generated in its interior through parthenogenesis would continue to fuel its fusion reactions. But in addition, there would be supplementation by energy spontaneously created in its interior through nonconservative photon blueshifting. As a result, a stellar core would not cool off, but would continue to radiate energy mainly in the form of $\mathrm{x}$-rays and cosmic rays.

This photon blueshifting prediction of Model G may be understood as follows. In supercritical regions of space, that is where $\varphi_{g}(r)<\varphi_{g c}$ photon energy will progressively increase in nonconservative fashion where Equation (16) now becomes expressed as:

$$
E(r)=E_{0} \mathrm{e}^{\beta r} .
$$

For $\varphi_{g}<0, \beta$ here takes on the role of an amplification coefficient, in effect acting as a "negative Hubble constant" that dictates exponential energy increase rather than tired light energy decrease. Evidence that photons exhibit a negative Hubble constant when passing through the gravity well of a galaxy cluster or supercluster is discussed by LaViolette [91] [96]. This relation is similar to that described earlier for the SQK cosmological redshift prediction, but with an amplification coefficient of opposite sign. Since observation shows that the cosmological redshift does not occur continuously, but takes place in discrete quantized jumps as mentioned earlier, a discrete energy increase of form similar to that described in Equation (13) may be inferred for this cosmological blueshifting phenomenon, in which case Equation (20) would be written as:

$$
E(r)=E_{0} \mathrm{e}^{n \beta \Delta r} .
$$

The excess energy evolved from photon energy amplification is termed genic energy. The genic energy of a body of mass $M$ would be given as:

$$
L_{\text {gen }}=\mathrm{d} E / \mathrm{d} t=\mu H \approx-\alpha \bar{\varphi}_{g} \bar{C} M \bar{T}
$$

where $H$ represents the body's total heat capacity, given by the product of its average gravity potential $\bar{\varphi}_{g}$, mass $M$, average specific heat $\bar{C}$, and average internal temperature $\bar{T}$. Among other things, this photon blueshifting prediction has been found to successfully predict the planetary-stellar M-L relation [111]. The reader is here referred to LaViolette [91] for further information on the astronomical significance of this blueshifting effect.

According to the SQK paradigm, a stellar core is unable to collapse into a black hole singularity due to its continuous creation of genic energy; see $\mathrm{LaVi}$ olette [91], Section 9.9. For example, since $L_{\text {gen }}$, the rate of genic energy production depends on both gravity potential and temperature, as depicted in Equation (21), $L_{g e n}$ of a collapsing stellar core would scale according to $1 / R^{2}$, where $R$ is stellar radius. That is, assuming that $\varphi_{g}(r) \propto 1 / R$ and $T \propto 1 / R$, then $L_{\text {gen }} \propto 1 / R^{2}$. Furthermore, since the surface area of the collapsing core varies as 
$1 / R^{2}$, the genic energy radiation pressure per unit surface area opposing collapse would vary according to $1 / R^{4}$. The inward pull of gravity opposing this, however, would increase only as $1 / R^{2}$. Consequently, with radiation pressure increasing faster than the inward force of gravity, a point would be reached where a core would ultimately cease to collapse. Consequently, a singularity would be unable to form.

Another reason why a black hole would not be able to form in SQK is because according to this physics the gravitational field potential within a subatomic particle should taper to a zero gradient at the particle's center. Hence as particles within a collapsing stellar core were pressed increasingly close together, the gravitational force attracting them to one another would approach zero. It is also worth noting that stellar cores may be supported from collapse entirely by genic energy radiation pressure, rather than by electron degeneracy. Consequently, in the cosmology of SQK, a supermassive galactic core would likely be a nondegenerate stellar core, rather than a black hole.

As stars continue to proliferate, they would collectively form a star cluster, and at their center would lie the supermassive stellar core that had given birth to their lineage. By this time the mother star core would have grown to hundreds to thousands of solar masses and would be expelling a wind of ionized gas and cosmic ray particles. Based on Equations (19) and (20), it is evident that the rate of matter and energy creation, and hence the nonconformance with energy conservation, would be most extreme in such supermassive cores, which is compatible with the ideas of Jeans, Ambartsumian, Sérsic, and Arp as mentioned earlier. $\mathrm{SQK}$ refers to such stellar cores as mother stars, the name highlighting the characteristic that a mother star would serve as a galaxy's primary matter and energy birthing site, being the most supercritical region in a galaxy.

As the star cluster continues to proliferate and grow in size, it eventually turns into a dwarf elliptical galaxy with its less massive stars orbiting about the Mother star along a preferred orbital plane. As the Mother star grows further in mass and creates an increasingly supercritical internal environment, its occasional outbursts would become increasingly energetic. Upon reaching a mass of a hundred thousand solar masses or more, its outbursts would begin to resemble those seen to come from the cores of Seyfert galaxies. These would propel stars and gas outward, causing the dwarf elliptical galaxy to evolve into a compact spiral galaxy and over time into a mature spiral galaxy. This progressive evolution from dwarf elliptical, to S0 spiral to Sc spiral would match the galaxy evolution progression suggested by Jeans and Hubble. Some of the more violent outbursts would cause the Mother star to fission and spew out a part of itself as a star cluster or even as a core embryo that could grow into a dwarf daughter galaxy. Halton Arp [104] [105] has catalogued many examples of what appear to be such core ejections; also see Sérsic [103]. These daughter bodies would orbit the spiral galaxy, forming a star cluster halo around the galaxy as well as spawning satellite galaxy progeny, as is considered to be happening in our own Milky Way. Eventually, as a result of continued core ejections, the spiral galaxy develops a spher- 
ical shape and evolves into a giant elliptical galaxy [91].

\section{Conclusions}

Arp [104] has stated that the observational evidence against the big bang theory has become overwhelming and that in reality the theory has been toppled. This is also the conclusion of the present overview. As shown above, the no-evolution, tired light model makes a better fit than the expanding universe hypothesis when compared to the observational data of seven cosmology tests. Including the results of the radio galaxy differential number count test [2], the total number of tests favoring the no-evolution, static universe tired light model comes to nine. Also, it is concluded that the supernova light curve test of Goldhaber, et al. is flawed by selection effect biases. Studies of X-ray bursts, gamma ray bursts, and quasar variability show no evidence of time dilation with increasing redshift. So based on the current evidence, one may conclude that the universe must be cosmologically stationary. Furthermore, the finding that the cosmological redshift values are quantized introduces a serious challenge to the Doppler redshift interpretation. The tired light theory fares much better since discrete quantum energy transitions are commonly known to microphysics. Hence tired light photons may be assumed to lose energy in discrete quantum steps.

Of the tired light theories that have been proposed, most account only for the cosmological redshift phenomenon. They do not simultaneously provide a matter creation cosmology that may replace the big bang theory. The subquantum kinetics physics paradigm, on the other hand, predicts tired light photon energy loss in intergalactic space and also provides a mechanism for the continuous creation of matter. Furthermore, SQK has been shown to spontaneously produce matter rather than antimatter, something that the big bang theory fails to do. Also, this physics predicts that a galaxy's gravity potential field should begin to depart from a Newtonian decline at distances greater than about $3 \mathrm{kpc}$ and ultimately plateau to a finite local extragalactic gravity potential value. This not only provides an answer as to why the static universe does not spontaneously undergo gravitational collapse, but also, in accordance with the conclusions of MOND, makes it unnecessary to assume the presence of dark matter in galaxies. Finally, the SQK tired light relation, whose energy attenuation coefficient is gravity potential dependent, may provide a resolution to the Fingers-of-God effect. If the present tight grip on the First Law can be ever so slightly relinquished, a new era should await the future of physics.

\section{Conflicts of Interest}

The author declares no conflicts of interest regarding the publication of this paper.

\section{References}

[1] Hickson, P. and Adams, P.J. (1979) Evidence for Cluster Evolution from the q-z 
Relation. Astrophysical Journal, 234, L91-L95. https://doi.org/10.1086/183116

[2] LaViolette, P.A. (1986) Is the Universe Really Expanding? Astrophysical Journal 301, 544-553. https://doi.org/10.1086/163922

[3] Gott, R.J., Gunn, G.E., Schramm, D.N. and Tinsley, B.M. (1974) An Unbound Universe? Astrophysical Journal, 194, 543-553. https://doi.org/10.1086/153273

[4] Bahcall, N.A. and Fan, X. (1998) A Lightweight Universe? Proceedings of the National Academy of Sciences of the United States of America, 95, 5956-5959. https://doi.org/10.1073/pnas.95.11.5956

[5] Goldhaber, G., Groom, D.E., Kim, A., Aldering, G., Astier, P., Conley, A., et al. (2001) Timescale Stretch Parameterization of Type I-a Supernova $B$-Band Light Curves. Astrophysical Journal, 558, 359-368. https://doi.org/10.1086/322460

[6] Lopez-Corredoira, M. (2010) Angular Size Test on the Expansion of the Universe. International Journal of Modern Physics D, 19, 245-291.

https://doi.org/10.1142/S0218271810016397

[7] Miley, G. (1971) The Radio Structure of Quasars-A Statistical Investigation. Monthly Notices of the Royal Astronomical Society, 152, 477-490. https://doi.org/10.1093/mnras/152.4.477

[8] Kellermann, K.I. (1972) Radio Galaxies, Quasars, and Cosmology. Astronomical Journal, 77, 531-542. https://doi.org/10.1086/111314

[9] Ubachukwu, A.A. and Onuora, L.I. (1993) Radio Source Orientation and the Cosmological Interpretation of the Angular Size-Redshift Relation for Double-Lobed Quasars. Astrophysics \& Space Science, 209, 169-180.

https://doi.org/10.1007/BF00627439

[10] Hoyle, F. (1959) The Relation of Radio Astronomy to Cosmology. In: Bracewell, R.N., Ed., Radio Astronomy, IAU Symposium No. 9, Stanford University Press, Stanford, 529.

[11] Sandage, A. (1988) Observational Tests of World Models. Annual Reviews of Astronomy \& Astrophysics, 26, 561-630.

https://doi.org/10.1146/annurev.aa.26.090188.003021

[12] Kapahi, V.K. (1987) The Angular Size-Redshift Relation as a Cosmological Tool. In: Hewitt, A., Burbidge, G. and Fang, L.Z., Eds., Observational Cosmology, Vol. 124, Dordrecht, 251-266. https://doi.org/10.1007/978-94-009-3853-3_23

[13] Andrews, T.B. (2006) Derivation of the Hubble Redshift and the Metric in a Static Universe. AIP Conference Proceedings, 822, 123-143.

https://doi.org/10.1063/1.2189129

[14] Nabokov, N.V. and Baryshev, Y.V. (2008) Classical Cosmological Tests for Galaxies of the Hubble Ultra Deep Field. Astrophysical Bulletin, 63, 244-258. https://doi.org/10.1134/S1990341308030048

[15] Lerner, E. (2015) Surface Brightness of Galaxies and the Evidence against the Concordance Model. Paper Presented at the Observational Anomalies Challenging the Lambda-CDM Cosmological Model. Special Session 2, European Week of Astronomy and Space Science 2015, La Laguna, 22-26 June 2015, 1339.

[16] Totani, T., Yoshii, Y., Maihara, T., Iwamuro, F. and Motohara, K. (2001) Near-Infrared Faint Galaxies in the Subaru Deep Field: Comparing the Theory with Observations for Galaxy Counts, Colors, and Size Distributions to K 24.5. Astrophysical Journal, 559, 592-605. https://doi.org/10.1086/322338

[17] Wright, E.L. (1987) Source Counts in the Chronometric Cosmology. Astrophysical Journal, 313, 551-555. https://doi.org/10.1086/164996 
[18] Tolman, R. (1930) On the Estimation of Distances in a Curved Universe with a Non-Static Line Element. Proceedings of the National Academy of Sciences of the United States of America, 16, 511-520. https://doi.org/10.1073/pnas.16.7.511

[19] Hubble, E. (1936) Effects of Red Shifts on the Distribution of Nebulae. Astrophysical Journal, 84, 517-554. https://doi.org/10.1086/143782

[20] Lubin, L.M. and Sandage, A. (2001) The Tolman Surface Brightness Test for the Reality of the Expansion. I. Calibration of the Necessary Local Parameters. Astronomical Journal, 121, 2271-2288. https://doi.org/10.1086/320394

[21] Lubin, L.M. and Sandage, A. (2001) The Tolman Surface Brightness Test for the Reality of the Expansion. II. The Effect of the Point-Spread Function and Galaxy Ellipticity on the Derived Photometric Parameters. Astronomical Journal, 121, 2289-2300. https://doi.org/10.1086/320401

[22] Lubin, L.M. and Sandage, A. (2001) The Tolman Surface Brightness Test for the Reality of the Expansion. III. Hubble Space Telescope Profile and Surface Brightness Data for Early-Type Galaxies in Three High-Redshift Clusters. Astronomical Journal, 122, 1071-1083. https://doi.org/10.1086/322133

[23] Lubin, L.M. and Sandage, A. (2001) The Tolman Surface Brightness Test for the Reality of the Expansion. IV. A Measurement of the Tolman Signal and the Luminosity Evolution of Early-Type Galaxies. Astronomical Journal, 122, 1084-1103. https://doi.org/10.1086/322134

[24] Aguirre, A. (1999) Dust versus Cosmic Acceleration. Astrophysical Journal, 512, L19-L22. https://doi.org/10.1086/311862

[25] Aguirre, A. (1999) Intergalactic Dust and Observations of Type IA Supernovae. Astrophysical Journal, 525, 583-593. https://doi.org/10.1086/307945

[26] Goobar, A., Bergström, L. and Mörtsell, E. (2002) Measuring the Properties of Extragalactic Dust and Implications for the Hubble Diagram. Astronomy \& Astrophysics, 384, 1-10. https://doi.org/10.1051/0004-6361:20020002

[27] Rowan-Robinson, M. (2002) Do type Ia Supernovae Prove $\Lambda>0$ ? Monthly Notices of the Royal Astronomical Society, 332, 352-360. https://doi.org/10.1046/j.1365-8711.2002.05299.x

[28] Kristian, J., Sandage, A. and Westphal, J.A. (1978) The Extension of the Hubble Diagram. II. New Redshifts and Photometry of Very Distant Galaxy Clusters: First Indication of a Deviation of the Hubble Diagram from a Straight Line. Astrophysical Journal, 221, 383-394. https://doi.org/10.1086/156038

[29] Postman, M. and Lauer, T.R. (1995) Brightest Cluster Galaxies as Standard Candles. Astrophysical Journal, 440, 28-47. https://doi.org/10.1086/175245

[30] LaViolette, P. (2002) Letter Correspondence with A. Sandage, 12/4/01 and 2/28/02.

[31] Sandage, A. (2002) Letter Correspondence with P. LaViolette, 3/27/02.

[32] Lerner, E.J. (2006) Evidence for a Non-Expanding Universe: Surface Brightness Data from HUDF. AIP Conference Proceedings, 822, 60-74. https://doi.org/10.1063/1.2189123

[33] Crawford, D.F. (2011) Observational Evidence Favors a Static Universe. Journal of Cosmology, 13, 3875-3946. Eprint: arxiv: 1009.0953. 3875, arXiv: 1009.0953.

[34] López-Corredoira, M. (2017) Tests and Problems of the Standard Model in Cosmology. Foundations of Physics, 47, 711-768. https://doi.org/10.1007/s10701-017-0073-8

[35] Lerner, E.J., Falomo, R. and Scarpa, R. (2014) UV Surface Brightness of Galaxies from the Local Universe to $\mathrm{z}$ 5. International Journal of Modern Physics D, 23, Ar- 
ticle ID: 1450058. https://doi.org/10.1142/S0218271814500588

[36] Pahre, M.A., Djorgovski, S.G. and de Carvalho, R.R. (1996) A Tolman Surface Brightness Test for Universal Expansion and the Evolution of Elliptical Galaxies in Distant Clusters. Astrophysical Journal Letters, 456, L79-L82. https://doi.org/10.1086/309872

[37] Andrews, T.B. (2006) Falsification of the Expanding Universe Model. AIP Conference Proceedings, 822, 3-22. https://doi.org/10.1063/1.2189118

[38] Jaakkola, T., Moles, M. and Vigier, J.P. (1979) Empirical Status in Cosmology and the Nature of Redshifts. Astronomische Nachrichten, 300, 229-238.

https://doi.org/10.1002/asna.19793000503

[39] Geller, M.J. and Peebles, P.J.E. (1972) Test of the Expanding Universe Postulate. Astrophysical Journal, 174, 1-5. https://doi.org/10.1086/151462

[40] Marosi, L.A. (2014) Hubble Diagram Test of 280 Supernovae Redshift Data. Journal of Modern Physics, 5, 29-33. http://doi.org/10.4236/jmp.2014.51005

[41] Marosi, L.A. (2019) Extended Hubble Diagram on the Basis of Gamma Ray Bursts Including the High Redshift Range of $\mathrm{z}=0.0331-8.1$. International Journal of Astronomy \& Astrophysics, 9, 1-11. https://doi.org/10.4236/ijaa.2019.91001

[42] Sandage, A., Tammann, G.A., Saha, A., Reindl, B., Macchetto, F.D. and Panagia, N. (2006) The Hubble Constant: A Summary of the Hubble Space Telescope Program for the luminosity Calibration of Type Ia Supernovae by Means of Cepheids. $A s$ trophysical Journal, 653, 843-860. https://doi.org/10.1086/508853

[43] Tammann, G.A. and Reindl, B. (2013) Alan Sandage and the Distance Scale. Proceedings of the International Astronomical Union, 8, 13-25.

https://doi.org/10.1017/S1743921312021059

[44] Planck Collaboration, Aghanim, N., et al. (2020) Planck 2018 Results. VI. Cosmological Parameters. Astronomy \& Astrophysics, 641, Article No. A6, 67 p, arXiv: 1807.06209. https://doi.org/10.1051/0004-6361/201833910

[45] Freedman, W.L., et al. (2019) The Carnegie-Chicago Hubble Program. VIII. An Independent Determination of the Hubble Constant Based on the Tip of the Red Giant Branch. Astrophysical Journal, 882, 34 (29 p.).

https://doi.org/10.3847/1538-4357/ab2f73

[46] Riess, A.G., Casertano, S., Yuan, W., Macri, L.M. and Scolnic, D. (2019) Large Magellanic Cloud Cepheid standards Provide a 1\% Foundation for the Determination of the Hubble Constant and Stronger Evidence for Physics beyond $\Lambda$ CDM. Astrophysical Journal, 876, Article No. 85, 13 p. https://doi.org/10.3847/1538-4357/ab1422

[47] Lombriser, L. (2020) Consistency of the Local Hubble Constant with the Cosmic Microwave Background. Physics Letters B, 803, Article ID: 135303.

https://doi.org/10.1016/j.physletb.2020.135303

[48] Ding, Q., Nakama, T. and Wang, Y. (2020) A Gigaparsec-Scale Local Void and the Hubble Tension. Science China Physics, Mechanics \& Astronomy, 63, Article No. 290403. https://doi.org/10.1007/s11433-020-1531-0

[49] Sokolov, V.V. (2001) On the GRB Progenitors: Possible Consequences for Supernovae Connection with Gamma-Ray Bursts. Bulletin of the Special Astrophysical Observatory, 51, Article No. 38. arXiv: astro-ph/0107399.

[50] Kocevski, D. and Petrosian, V. (2013) On the Lack of Time Dilation Signatures in Gamma-Ray Burst Light Curves. Astrophysical Journal, 765, Article No. 116, 7 p. arXiv: 1110.6175. https://doi.org/10.1088/0004-637X/765/2/116 
[51] Crawford, D.F. (2009) No Evidence of Time Dilation in Gamma-Ray Burst Data. arXiv: 0901.4169.

[52] Hawkins, M. (2010) On Time Dilation in Quasar Light Curves. Monthly Notices of the Royal Astronomical Society, 405, 1940-1946.

https://doi.org/10.1111/j.1365-2966.2010.16581.x

[53] Phillips, M.M. (1993) The Absolute Magnitudes of Type IA Supernovae. Astrophysical Journal, 413, L105-L108. https://doi.org/10.1086/186970

[54] Hamuy, M., Phillips, M.M., Suntzeff, N.B., Schommer, R.A., Maza, J., Smith, R.C., et al. (1996) The Morphology of Type IA Supernovae Light Curves. Astronomical Journal, 112, 2438-2447. https://doi.org/10.1086/118193

[55] Quimby, R.M., Kulkarni, S.R., Kasliwal, M.M., Gal-Yam, A., Arcavi, I., Sullivan, M., et al. (2011) Hydrogen-Poor Super-Luminous Stellar Explosions. Nature, 474, 487-489. https://doi.org/10.1038/nature10095

[56] Crawford, D.F. (1999) Curvaturepressurein a Cosmology with a Tired-Light Redshift. Australian Journal of Physics, 52, 753-777.

https://doi.org/10.1071/PH98065

[57] Masreliez, J. (2002) Accelerating Universe or Tired Light?

[58] Tifft, W.G. (1976) Discrete States of Redshift and Galaxy Dynamics. I. Internal Motions in Single Galaxies. Astrophysical Journal, 206, 38-56.

https://doi.org/10.1086/154354

[59] Tifft, W.G. (1978) The Absolute Solar Motion and the Discrete Redshift. Astrophysical Journal, 221, 756-775. https://doi.org/10.1086/156079

[60] Tifft, W.G. (1980) Periodicity in the Redshift Intervals for Double Galaxies. Astrophysical Journal, 236, 70-74. https://doi.org/10.1086/157719

[61] Tifft, W.G. (1982) Quantum Effects in the Redshift Intervals for Double Galaxies. Astrophysical Journal, 257, 442-449. https://doi.org/10.1086/160002

[62] Tifft, W.G. (1982) Double Galaxy Investigations. II. The Redshift Periodicity in Optically Observed Pairs. Astrophysical Journal, 262, 44-47.

https://doi.org/10.1086/160394

[63] Cocke, W.J. and Tifft, W.G. (1983) A Theoretical Framework for Quantized Redshifts and Uncertainty in Cosmology. Astrophysical Letters, 23, 239-249.

[64] Cocke, W.J. and Tifft, W.G. (1983) Redshift Quantization in Compact Groups of Galaxies. Astrophysical Journal, 268, 56-59. https://doi.org/10.1086/160928

[65] Tifft, W.G. and Cocke, W.J. (1984) Global Redshift Quantization. Astrophysical Journal, 287, 492-502. https://doi.org/10.1086/162708

[66] Tifft, W.G. (1991) Properties of the Redshift III: Temporal Variation. Astrophysical Journal, 382, 396-415. https://doi.org/10.1086/170730

[67] Guthrie, B.N.G. and Napier, W. (1996) Redshift Periodicity in the Local Supercluster. Astronomy \& Astrophysics, 310, 353-370.

[68] Napier, W. and Guthrie, B.N.G. (1997) Quantized Redshifts: A Status Report. Journal of Astrophysics \& Astronomy, 18, 455-463. https://doi.org/10.1007/BF02709337

[69] Narlikar, J. (1977) Two Astrophysical Applications of Conformal Gravity. Annals of Physics, 107, 325-336. https://doi.org/10.1016/0003-4916(77)90214-7

[70] Arp, H. (1987) Quasars, Redshifts, and Controversies. Cambridge University Press, Cambridge. https://doi.org/10.1017/CBO9780511564857

[71] Arp, H. (1986) A Corrected Velocity for the Local Standard of Rest by Fitting to the 
Mean Redshift of Local Group Galaxies. Astronomy \& Astrophysics, 156, 207-212.

[72] Broadhurst, T.J., Ellis, R.S., Koo, D.C. and Szalay, A.S. (1990) Large-Scale Distribution of Galaxies at the Galactic Poles. Nature, 343, 726-728.

https://doi.org/10.1038/343726a0

[73] Oesch, P.A., Brammer, G., Brammer, G., van Dokkum, P.G., Illingworth, G.D., Bouwens, R.J., et al. (2016) A Remarkably Luminous Galaxy at $\mathrm{Z}=11.1$. Measured with Hubble Space Telescope Grism Spectroscopy. Astrophysical Journal, 819, Article No. 129, arXiv: 1603.00461. https://doi.org/10.3847/0004-637X/819/2/129

[74] Ellis, R.S., McLure, R.J., Dunlop, J.S., Robertson, B.E., Ono, Y., Schenker, M., A., et al. (2013) The Abundance of Star-Forming Galaxies in the Redshift Range 8.5 to 12: New Results from the 2012 Hubble Ultra Deep Field Campaign. Astrophysical Journal Letters, 763, Article No. L7, arXiv: 1211.6804.

https://doi.org/10.1088/2041-8205/763/1/L7

[75] Zwicky, F. (1929) On the Red Shift of Spectral Lines through Interstellar Space. Proceedings of the National Academy of Sciences of the United States of America, 15, 773-779. https://doi.org/10.1073/pnas.15.10.773

[76] Pecker, J.-C. and Vigier, J.-P. (1987) A Possible Tired-Light Mechanism. Proceedings of the 124th Symposium of the International Astronomical Union, Beijing, 25-30 August 1986, 507-511. https://doi.org/10.1007/978-94-009-3853-3_48

[77] Marmet, P. (1981) A New Non-Doppler Redshift. Universite Laval, Department of Physics, Quebec, 64 p.

[78] Marmet, P. (1988) A New Non-Doppler Redshift. Physics Essays, 1, 24-32. https://doi.org/10.4006/1.3033412

[79] Marmet, P. and Reber, G. (1989) Cosmic Matter and the Nonexpanding Universe. IEEE Transactions of Plasma Science, 17, 264-269. https://doi.org/10.1109/27.24634

[80] Zheng, Y. (2013) The Propagation of Photons in the Dilute Ionized Gas. arXiv: 1305.0427.

[81] Maxwell, J.C. (1954) A Treatiseon Electricity and Magnetism. Vol. II, Dover, New York.

[82] Monti, R. (1988) The Electric Conductivity of Background Space. In: Kostro, L., Posiewnik, A., Pykacz, J. and Zukowski, M., Eds., Problems in Quantum Physics, Gdansk87, World Scientific, Teaneck, 640.

[83] von Nernst, W. (1921) The Structure of the Universe in Light of Our Research. Jules Springer, Berlin.

[84] von. Nernst, W. (1938) Additional Test of the Assumption of a Stationary State in the Universe. Zeitschrift fur Physik, 106, 633-661.

https://doi.org/10.1007/BF01339902

[85] Monti, R. (1986) Albert Einstein and Walther Nernst: Comparative Cosmology. SeaGreen, 4, 32-36.

[86] Vigier, J.-P. (1990) Evidence for Nonzero Mass Photons Associated with a Vacuum-Induced Dissipative Red-Shift Mechanism. IEEE Transactions of Plasma Science, 18, 64-72. https://doi.org/10.1109/27.45506

[87] LaViolette, P.A. (1985) An Introduction to Subquantum Kinetics. III. The Cosmology of Subquantum Kinetics. International Journal of General Systems, 11, 329-345. https://doi.org/10.1080/03081078508934920

[88] LaViolette, P.A. (1985) An Introduction to Subquantum Kinetics. I. An Overview of the Methodology. International Journal of General Systems, 11, 281-293.

https://doi.org/10.1080/03081078508934918 
[89] LaViolette, P.A. (1985) An Introduction to Subquantum Kinetics. II. An Open Systems Description of Particles and Fields. International Journal of General Systems, 11, 295-328. https://doi.org/10.1080/03081078508934919

[90] LaViolette, P.A. (2008) The Electric Charge and Magnetization Distribution of the Nucleon: Evidence of a Subatomic Turing Wave Pattern. International Journal of General Systems, 37, 649-676. https://doi.org/10.1080/03081070802367457 https://starburstfound.org/downloads/physics/nucleon.pdf

[91] LaViolette, P.A. (2012) Subquantum Kinetics: A Systems Approach to Physics and Cosmology. 4th Edition, Starlane Publications, Niskayuna.

[92] LaViolette, P.A. (2012) The Cosmic Ether: Introduction to Subquantum Kinetics. Physics Procedia, 38, 326-349. https://doi.org/10.1016/j.phpro.2012.08.032

[93] Pulver, M. and LaViolette, P.A. (2013) Stationary Dissipative Solitons of Model G. International Journal of General Systems, 42, 519-541. https://doi.org/10.1080/03081079.2013.776313

[94] Gmitro, J.I. and Scriven, L.E. (1966) A Physiochemical Basis for Pattern and Rhythm. In: Warren, K., Ed., Intracellular Transport, Academic Press, New York, 221-255. https://doi.org/10.1016/B978-1-4831-9872-9.50016-0

[95] LaViolette, P.A. (2021) The Origin of Gravity and Its Effects: According to the Subquantum Kinetics Paradigm. In: Krasnoholovets, V., Ed., The Origin of Gravity from the First Principles, Nova Science Publishers, Hauppauge. (In Press) https://starburstfound.org/downloads/physics/Origin-Gravity-Its-Effects.pdf

[96] LaViolette, P.A. (2021) The Nonvelocity Spectral Shift. Submitted.

[97] Jeans, J. (1919) Problems of Cosmogony and Stellar Dynamics. Cambridge University Press, London.

[98] Jeans, J. (1928) Astronomy and Cosmogony. Cambridge University Press, London.

[99] Hubble, E.P. (1926) Extragalactic Nebulae. Astrophysical Journal, 64, 321-369. https://doi.org/10.1086/143018

[100] McCrea, W.H. (1964) Continual Creation. Monthly Notices of the Royal Astronomical Society, 128, 335-344. https://doi.org/10.1093/mnras/128.4.335

[101] Ambartsumian, V.A. (1958) On the Evolution of Galaxies. Proceedings of the 11th Solvay Conference. Structure and Evolution of the Universe, Solvay, 1958, Institute Internationale de Physique, 241-249.

[102] Ambartsumian, V.A. (1965) On the Nuclei of Galaxies and Their Activity. Proceedings of the 13 th Solvay Conference on Physics. The Structure and Evolution of Galaxies, Brussels, September 1964, Interscience Publishers, London, 1-16.

[103] Sérsic, J.L. (1968) On the Formation of Galaxies by Fragmentation. Bulletin of the Astronomical Institute of Czechoslovakia, 19, 105-110.

[104] Arp, H. (1998) The Origin of Companion Galaxies. Astrophysical Journal, 496, 661-669. https://doi.org/10.1086/305412

[105] Arp, H., Burbidge, E.M., Chu, Y. and Zhu, X. (2001) X-Ray-Emitting QSOs Ejected from Arp 220. Astrophysical Journal, 553, L11-L13. https://doi.org/10.1086/320499

[106] LaViolette, P.A. (1994) The Matter of Creation. Physics Essays, 7, 1-8. https://starburstfound.org/downloads/physics/MOC.pdf

[107] Kelly, J.J. (2002) Nucleon Charge and Magnetization Densities from Sachs form Factors. Physical Review C, 66, Article ID: 065203.

https://doi.org/10.1103/PhysRevC.66.065203

[108] Burbidge, G. and Hoyle, F. (1998) The Origin of Helium and Other Light Elements. 
Astrophysical Journal, 509, L1-L3. https://doi.org/10.1086/311756

[109] Crawford, D.F. (1987) Diffuse Background X Rays and the Density of the Intergalactic Medium. Australian Journal of Physics, 40, 459-464.

https://doi.org/10.1071/PH870459

[110] Arp, H., Burbidge, G., Hoyle, F. Wickramasinghe, N. and Narlikar, J. (1990) The Extragalactic Universe: An Alternative View. Nature, 346, 807-812.

https://doi.org/10.1038/346807a0

[111] LaViolette, P.A. (1992) The Planetary-Stellar Mass-Luminosity Relation: Possible Evidence of Energy Nonconservation? Physics Essays, 5, 536-543. 